\title{
Hypermetabolic Cerebellar Connectome in Alzheimer's Disease
}

\author{
by \\ Vinay Gupta \\ A Thesis submitted to Faculty of Graduate Studies of \\ The University of Manitoba \\ In partial fulfillment of the requirements of the degree of
}

MASTER OF SCIENCE

Graduate Program in Biomedical Engineering

University of Manitoba

Winnipeg

Copyright $\odot 2020$ by Vinay Gupta 


\begin{abstract}
:
(for the Alzheimer's Disease Neuroimaging Initiative*)
\end{abstract}

Alzheimer's disease (AD) is typically characterized by hypometabolism in medial-frontal lobes, posterior cingulum and temporal lobes which can be assessed using fluorodeoxyglucose (FDG) positron emission tomography (PET) in vivo. Hypermetabolism in cerebellum and pons have been also consistently reported, but it is often neglected. Some studies have dismissed it as an artifact of global mean normalization. We hypothesize that the hypermetabolic regions are also important in disease pathology in AD. Using 88 AD subjects and 88 age-sex matched Normal Controls (NL1) from the publicly available neuroimaging database enabled by Alzheimer's disease Neuroimaging Initiative (ADNI; http://adni.loni.usc.edu/), we established FDG PET-based AD Classifier (FAC) that differentiated AD patients from normal individuals (sensitivity $=87.50 \%$, specificity $=82.95 \%$ ). To rule out that the observed hypermetabolism is an artifact induced by global signal intensity normalization, we replicated FAC using white matter (WM) as a reference region, and we confirmed the presence of hypermetabolism in cerebellum and pons. The brain metabolic network of AD and NL has been further analyzed using graph theory. The differences in Betweenness Centrality (BC, a measure 
of hubness of information flow) between AD vs NL network were correlated with region weights of FAC, which was driven by a few brain regions. In particular, the hypermetabolism in cerebellum was accompanied with higher $\mathrm{BC}$ while the hypometabolism in temporal lobe and posterior cingulum were accompanied with lower $\mathrm{BC}$ in $\mathrm{AD}$. The brain regions with higher $\mathrm{BC}$ in $\mathrm{AD}$ network showed a progressive increase in FDG uptake (hypermetabolism) over 2 years in prodromal AD patients $(n=39)$, whose baseline scans were acquired at least 2 years prior to the AD diagnosis. This progressive increase of FDG uptake was not observed in the control subjects who had mild cognitive impairment but did not develop AD over the next 2 years $(n=39)$ nor in NL who were followed up for 2 years $(n=39)$. This study suggests that hypermetabolism in the cerebellum associated with AD may play an important role in forming the AD-related metabolic network. 
*Data used in preparation of this article were obtained from the Alzheimer's Disease Neuroimaging Initiative (ADNI) database (adni.loni.usc.edu). As such, the investigators within the ADNI contributed to the design and implementation of ADNI and/or provided data but did not participate in analysis or writing of this report.

A complete listing of ADNI investigators can be found at: http://adni.loni.usc.edu/wp-

content/uploads/how to apply/ADNI Acknowledgement List.pdf 


\section{Acknowledgments}

I would like to take this opportunity to express my sincere gratitude to my supervisor Dr. Ji Hyun Ko. Without Dr. Ko's careful guidance, expertise and constant support at each step of my project, this project would not be possible. I would like to extend my gratitude to the members of my advisory committee, Dr. Zahra Moussavi and Dr. Andrew Goertzen; who despite their busy schedules, have given their valuable time to evaluate my research work and offered their critiques and advice along the way. I would like to thank Samuel Booth from the Ko lab, who has been a crucial help to me in the process of drafting my manuscript. I would like to acknowledge and thank Dr. Dali Zhang for his help in getting set up with my project. I also need to thank my lab members Natalie Wright and Eun Choi, who have provided their valuable feedback on my research and often shared suggestions for improving my presentation skills. I would also like to thank Kae Normandeau and Crystal Acosta for their help in getting WHMIS training.

This project would not have been possible without access to a freely available online neuroimaging database which ADNI has provided. I need to express my sincere gratitude to ADNI for providing access to the database so that I could 
work on this project. I would like to thank my funding agencies Mitacs and NSERC, who have provided help with graduate scholarships and project funding respectively. The financial assistance from these agencies made it feasible for me to pursue this project. I express my sincere thanks to my family and friends, who have been a constant pillar of support during hard times. I need to give a special shout out to Ko Lab and all my colleagues-friends with whom I shared these last two years working on this project.

Data collection and sharing for this project was funded by the Alzheimer's Disease Neuroimaging Initiative (ADNI) (National Institutes of Health Grant U01 AG024904) and DOD ADNI (Department of Defense award number W81XWH-12-2-0012). ADNI is funded by the National Institute on Aging, the National Institute of Biomedical Imaging and Bioengineering, and through generous contributions from the following: AbbVie, Alzheimer's Association; Alzheimer's Drug Discovery Foundation; Araclon Biotech; BioClinica, Inc.; Biogen; Bristol-Myers Squibb Company; CereSpir, Inc.; Cogstate; Eisai Inc.; Elan Pharmaceuticals, Inc.; Eli Lilly and Company; EuroImmun; F. HoffmannLa Roche Ltd and its affiliated company Genentech, Inc.; Fujirebio; GE Healthcare; IXICO Ltd.; Janssen Alzheimer Immunotherapy Research \& Development, LLC.; Johnson \& Johnson Pharmaceutical Research \& 
Development LLC.; Lumosity; Lundbeck; Merck \& Co., Inc.; Meso Scale Diagnostics, LLC.; NeuroRx Research; Neurotrack Technologies; Novartis Pharmaceuticals Corporation; Pfizer Inc.; Piramal Imaging; Servier; Takeda Pharmaceutical Company; and Transition Therapeutics. The Canadian Institutes of Health Research is providing funds to support ADNI clinical sites in Canada. Private sector contributions are facilitated by the Foundation for the National Institutes of Health (www.fnih.org). The grantee organization is the Northern California Institute for Research and Education, and the study is coordinated by the Alzheimer's Therapeutic Research Institute at the University of Southern California. ADNI data are disseminated by the Laboratory for Neuro Imaging at the University of Southern California. 


\section{Table of Contents}

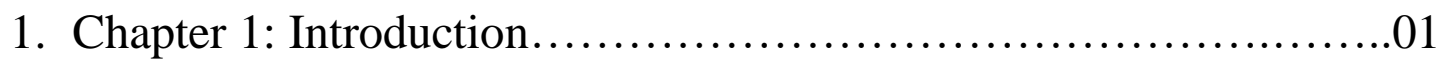

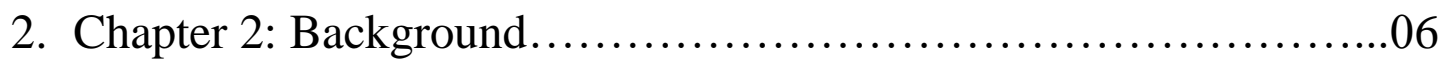

2.1. Alzheimer's Disease...................................06

2.2. Pathophysiology of Alzheimer's Disease..................08

2.3. Clinical Expression...................................12

2.4. Current State of Diagnosis..............................14

2.5. Treatment of Alzheimer's Disease.......................17

2.6. Overview of PET Imaging................................19

2.7. Factors influencing FDG uptake in AD ...................21

2.8. Alzheimer's Disease Neuroimaging Initiative (ADNI)..23

2.9. Graph Theory .........................................26

2.10. Application of Graph Theory ...........................29

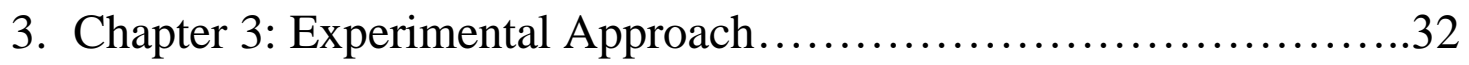

3.1. Subjects............................................ 32

3.2. Image Acquisition.................................... 38

3.3. Image Processing ...........................................

3.4. FDG PET-based AD Classifier (FAC) ..................40

3.5. AD Network analysis using Graph Theory ...............42

3.6. Longitudinal Changes in FDG SUR .....................44

4. Chapter 4: Results..................................................46

4.1. FDG PET-based AD Classifier (FAC) ...................46

4.2. AD Metabolic Network Profile............................48

4.3. Hubness correlate with abnormal AD metabolic topography .............................................

4.4. Longitudinal Changes of FDG-SUR in Hub vs Non-Hub

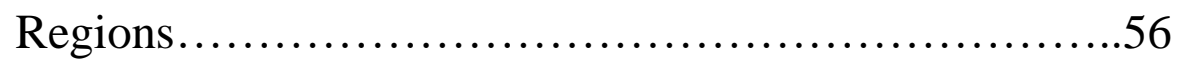

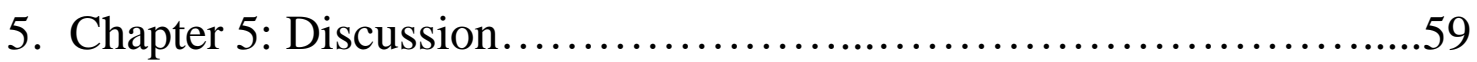

6. Chapter 6: Conclusion................................................68

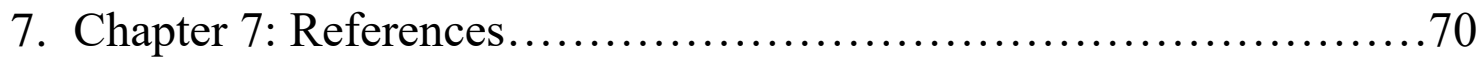




\section{List of Tables}

1. Table 1: Demographic data for ADNI subjects......................34 


\section{List of Figures}

\begin{tabular}{|l|l|}
\hline Figure 1: & $\begin{array}{l}\text { The sequence of pathogenic events as outlined by } \\
\text { amyloid cascade hypothesis }\end{array}$ \\
\hline Figure 2: & Subject Selection Protocol for ADNI subjects \\
\hline Figure 3: & FDG PET-based AD Classifier (FAC) \\
\hline Figure 4: & $\begin{array}{l}\text { Changes in Global network metrics in AD network and } \\
\text { NL network as a function of network cost }\end{array}$ \\
\hline Figure 5: & $\begin{array}{l}\text { Correlation between FAC Region Weights and } \\
\text { Betweenness Centrality of regions }\end{array}$ \\
\hline Figure 6: & $\begin{array}{l}\text { Effect of Brain Parcellation Scheme on Relationship } \\
\text { between ADRP Region Weights and Betweenness } \\
\text { Centrality }\end{array}$ \\
\hline Figure 7: & Longitudinal Changes in FDG-SUR \\
\hline
\end{tabular}


Table of Abbreviations

\begin{tabular}{|c|c|}
\hline Abbreviation & Definition \\
\hline AAL & Automated Anatomical Labelling \\
\hline $\mathrm{AD}$ & Alzheimer's Disease \\
\hline ADNI & $\begin{array}{l}\text { Alzheimer's Disease Neuroimaging } \\
\text { Initiative }\end{array}$ \\
\hline APP & Amyloid Precursor Protein \\
\hline $\mathrm{A} \beta$ & Amylod Beta \\
\hline $\mathrm{BC}$ & Betweenness Centrality \\
\hline $\mathrm{C}$ & Clustering Coefficient \\
\hline CDR & Clinical Dementia Rating \\
\hline CMRglc & Cerebral Metabolic Rate of Glucose \\
\hline CSF & Cerebrospinal Fluid \\
\hline DLB & Demetian with Lewy Body \\
\hline $\mathrm{DMN}$ & Default Mode Network \\
\hline FAC & FDG PET-based AD Classifier \\
\hline FAQ & Functional Assessment Questionnaire \\
\hline FDG & Fluorodeoxyglucose \\
\hline fMRI & Functional Magnetic Resonance Imaging \\
\hline FTD & Frontotemporal Dementia \\
\hline GM & Grey Matter \\
\hline GT & Graph Theory \\
\hline KP & Kisspeptine \\
\hline $\mathrm{L}$ & Characteristic Path Length \\
\hline LONI & Laboratory of Neuroimaging \\
\hline MCI & Mild Cognitive Impairment \\
\hline MMSE & Mini Mental State Examination \\
\hline MNI & Montreal Neuroimaging Initiative \\
\hline $\mathrm{MoCA}$ & Montreal Cognitive Assessment \\
\hline MRI & Magnetic Resonance Imaging \\
\hline NL & Normal Controls \\
\hline
\end{tabular}




\begin{tabular}{|c|c|}
\hline PAD & Prodromal Alzheimer's Disease \\
\hline PET & Positron Emission Tomography \\
\hline RM-ANOVA & Repeated Measures-Analysis of Variance \\
\hline ROI & Region of Interest \\
\hline RS & Resting State \\
\hline SMCI & Stable Mild Cognitive Imapirment \\
\hline SPM 12 & Statistical Parametric Mapping 12 \\
\hline SUR & Standard Uptake Ratio \\
\hline SUV & Standard Uptake Value \\
\hline WM & White Matter \\
\hline
\end{tabular}




\section{Chapter 1: Introduction:}

Alzheimer's Disease (AD) is the most common form of dementia affecting 5.8 million people in the United States alone (1). AD has accounted for 110,561 deaths in 2015 in United States, which makes AD the sixth leading cause of death in the United States (2). By 2050, the number of people living with AD dementia in the United States may grow to 13.8 million on account of the aging baby-boom generation (3).

$\mathrm{AD}$ is a neurodegenerative disorder marked by an irreversible, progressive decline in cognitive capabilities leading to the deterioration of memory and thinking skills, which eventually results in the loss of ability to carry out the simplest tasks. Studies have established that AD pathology begins 20 years or more before symptoms arise, with small changes in the brain that are unnoticeable to the person (4). The accumulation of beta-amyloid plaques and tau tangles are associated with AD pathophysiology (5).

Fluorodeoxyglucose (FDG) positron emission tomography (PET) signal has often been used to approximate synaptic activity (6); thus, it has been commonly accepted as an imaging biomarker sensitive to cognitive decline in $\mathrm{AD}(7) . \mathrm{AD}$ is typically characterized by hypometabolism in medial-frontal lobes, posterior cingulum and temporal lobes when compared to age-gender 
matched normal controls $(8,9)$. Yet, due to significant variation in patient PET signal expression, clinical reading of FDG PET images is usually based on the subjective impression of relative hypo-metabolism (10). The subjective nature of FDG-PET readings limit the use of a biomarker-related definitive threshold of FDG uptake in AD diagnosis (7).

In recent times, there has been an increased focus to develop better biomarkers to aid in AD diagnosis, e.g., the Alzheimer's Disease Neuroimaging Initiative (ADNI) is a longitudinal multicenter study involving combined efforts of multiple research centers across North America (http://adni.loni.usc.edu/about/). ADNI has collected the largest available multi-modal longitudinal brain imaging database for patients with $\mathrm{AD}$, mild cognitive impairment (MCI) and normal control subjects (11). Utilizing the ADNI database, several studies have established quantitative biomarkers that may aid in $\mathrm{AD}$ diagnosis. Regardless of the type of pattern recognition approach, multi-modal prediction model studies suggest that FDG PET is one of the most relevant imaging modalities that aided AD classification $(9,10,12-$ $15)$.

Previous neuroimaging studies have consistently reported a decline in FDG uptake within medial-frontal lobes, temporo-parietal and posterior 
cingulum cortices in $\operatorname{AD}(7,9)$. Recent studies have also reported increased FDG uptake in cerebellum and pons (8). Despite being consistently reported in various studies, hypermetabolism observed in $\mathrm{AD}$ is often neglected in $\mathrm{AD}$ diagnosis $(16,17)$.

Some studies have suggested that hypermetabolism is observed in neurodegenerative disorders on account of different signal intensity normalization procedure (e.g., global mean normalization) (17). In addition, these hypermetabolic regions (e.g., cerebellum and pons) are not traditionally perceived as key anatomical structures that are affected by $\mathrm{AD}$; although recent studies demand reconsideration of their positions $(18,19)$. For example, it has been suggested that the cerebellum has an integral contribution to cognitive and neuropsychiatric deficits in AD (18). Neuroprotective compounds such as hormone Kisspeptine (KP) have been shown to be neuroprotective against $A \beta$ toxicity in pons of $\mathrm{AD}$ brain (20) and might explain increased metabolism observed in pontine region in $\mathrm{AD}$ subjects.

Graph theory is an analytic approach that investigates the hierarchy of nodal structure within a network, e.g., identifying the hub of the information transfer $(21,22)$. With anatomical parcellation scheme and group-wise regionto-region correlation map, one can investigate the brain metabolic network 
topology associated with neurodegenerative disorders (23). Studies have shown brain network connectivity to be altered and impaired in AD patients partly due to increased amyloid plaque pathology (24).

Using graph theory analysis on the brain metabolic network, a previous study revealed an important contribution of hypermetabolic anatomical structures including the cerebellum in the pathological network formulation in Parkinson's disease (25), the hypermetabolism of which has been similarly disregarded, previously. To our knowledge, this has not been explicitly investigated in $\mathrm{AD}$. To address this issue, we explored the topographical relationship between the disease-related metabolic status (hypermetabolism vs. hypometabolism) of different regions identified in the FDG PET-based AD Classifier (FAC) and their functional hubness measured by Betweenness Centrality (BC) (25).

In the current study, we investigated the role of hypermetabolic regions such as cerebellum in AD metabolic network. We validated the relevance of hypermetabolism observed in $\mathrm{AD}$ patients using White Matter mean for signal normalization. Using $\mathrm{BC}$ as a measure of hubness of the regions, we investigated the significance of each region in the information flow network on 
AD patients. We also examined the longitudinal metabolic changes via FDG uptake in regions with increased hubness in $\mathrm{AD}$ metabolic network.

To validate if our results are dependent on different brain parcellation approach, the analysis has been repeated with two different parcellation schemes, automated anatomical labeling (AAL, the most widely used brain parcellation method) (26) and 268-node functional atlas generated via groupwise spectral clustering (an atlas based on functional homogeneity within each subunit) $(27,28)$. 


\section{Chapter 2: Background}

In this chapter, a review on the basics and existing literarture on various components of study is provided.

\subsection{Alzheimer's Disease}

AD was first identified by German psychiatrist Aloïs Alzheimer in 1906 (29), but about 70 years passed before it was recognized as a common cause of dementia and major cause of death (30). As of 2015, the economic burden of AD exceeds USD 818 million per year (31). The decline in cognitive abilities in patients suffering from $\mathrm{AD}$ is a difficult burden on patients, families and caregivers.

The symptoms of dementia due to Alzheimer's vary among people over a wide array of cognitive deficiencies such as memory loss, personality and behavioral changes and difficulty in performing daily tasks. The neurological changes taking place in the brain due to Alzheimer's pathology precede the onset of symptoms; changes at the molecular level are thought to begin 20 years or more before cognitive changes become detectable (32). There are no simple and inexpensive tests available for $\mathrm{AD}$ diagnosis which could be used reliably.

Early detection of $\mathrm{AD}$ improves the treatment outcome by allowing early 
intervention (3), and subsequent psychosocial education allows patients and caregivers an opportunity to plan better for the future (1).

With the exception of familial Alzheimer's disease which is linked to genetic mutation, it is believed that $\mathrm{AD}$ develops as a result of multiple risk factors. The primary risk factor for AD remains old age with high incidence reported in individuals older than 65 years (33). AD is also associated with risk factors such as education, cardiovascular health, social and cognitive engagement; which can be changed to reduce the risk of cognitive decline and dementia. Some researchers believe that having more years of formal education builds the 'cognitive reserve' of an individual. Cognitive reserve is the brain's ability to utilize pre-existing cognitive networks to enable a person to carry out cognitive tasks despite the changes in brain.

Cognitive reserve is also shown to be more robust in individuals with mentally stimulating jobs or who are more frequently engaged in mentally stimulating activities (34). Studies suggest that remaining socially and mentally engaged throughout life contributes to cognitive reserve in an individual and helps in reducing the risk of Alzheimer's and other dementias (35). More research needs to be conducted to better understand the role of social and cognitive engagement in reducing the risk of dementia due to Alzheimer's (34). 
Neuroprotective factors such as maintaining a low dietary intake of saturated fats and engaging in mentally stimulating activities and exercises such as learning new languages have been shown to decrease the overall risk of developing $\mathrm{AD}(35)$.

\subsection{Pathophysiology of Alzheimer's Disease}

The prominent pathological characteristic of $\mathrm{AD}$ is the formation and accumulation of abnormally folded protein fragments called beta-amyloid (plaques) outside neurons in the brain and twisted strands of protein tau (tangles) inside neurons (36). Post-mortem studies of AD patients have revealed that $\mathrm{A} \beta$ deposition is abundantly distributed in the cortex early in the course of AD progression, whereas tau formation, synaptic loss and gliosis progress to advanced stages of $\mathrm{AD}$ (37). The exact causes of $\mathrm{AD}$ are only known in cases of familial Alzheimer's disease, which have a clear genetic link.

The relationship between amyloid plaques, neurofibrillary tangles, and dementia is not very well understood in sporadic AD (38). Several hypotheses have been proposed for $\mathrm{AD}$ based on different causative factors to explain the multifactorial nature of $\mathrm{AD}$ (39) such as the beta-amyloid hypothesis, cholinergic hypothesis, tau hypothesis, and inflammation hypothesis (38). One of the most prevalent pathophysiologic hypotheses for senile plaque formation 
in $\mathrm{AD}$ is the amyloid cascade hypothesis which suggests that the primary cause for Alzheimer's dementia is related to the accumulation of amyloid-beta protein (36).

As per the amyloid cascade hypothesis, the amyloid precursor protein (APP) in brain is cleaved by $\alpha$-secretase and atypically processed by $\beta$ - and $\gamma$ secretases resulting in formation of amyloid beta (38). This process causes an imbalance between production and removal of beta-amyloid peptides resulting in accumulation of beta-amyloid peptides into soluble oligomers and they are eventually deposited in diffuse senile plaques (39). The presence of congophilic $\mathrm{A} \beta$-enriched plaques in the brains of $\mathrm{AD}$ patients revealed through post-mortem studies encourages the amyloid cascade hypothesis (36). The sequence of pathogenic events leading to $\mathrm{AD}$ (5) as proposed by the amyloid cascade hypothesis are shown in figure 1. The gradual accumulation of beta-amyloid plaques in our brain with aging suggests the presence of a threshold concentration of beta-amyloid beyond which they become toxic and results in further symptoms of $\mathrm{AD}(40)$.

Accumulation of neurofibrillary tau tangles inside the neurons, which are comprised of tau proteins, is another characteristic feature of AD (41). The balance between the phosphorylation of tau by kinase proteins and 
dephosphorylation by phosphatase proteins determines the level of phosphorylation in target tau molecules. In $\mathrm{AD}$, protein phosphatase $2 \mathrm{~A}$ (PP2A) is found $20-30 \%$ less active (42). The presence of neurofibrillary tangle in AD patients increases in parallel with the severity and duration of $\mathrm{AD}(41)$. 


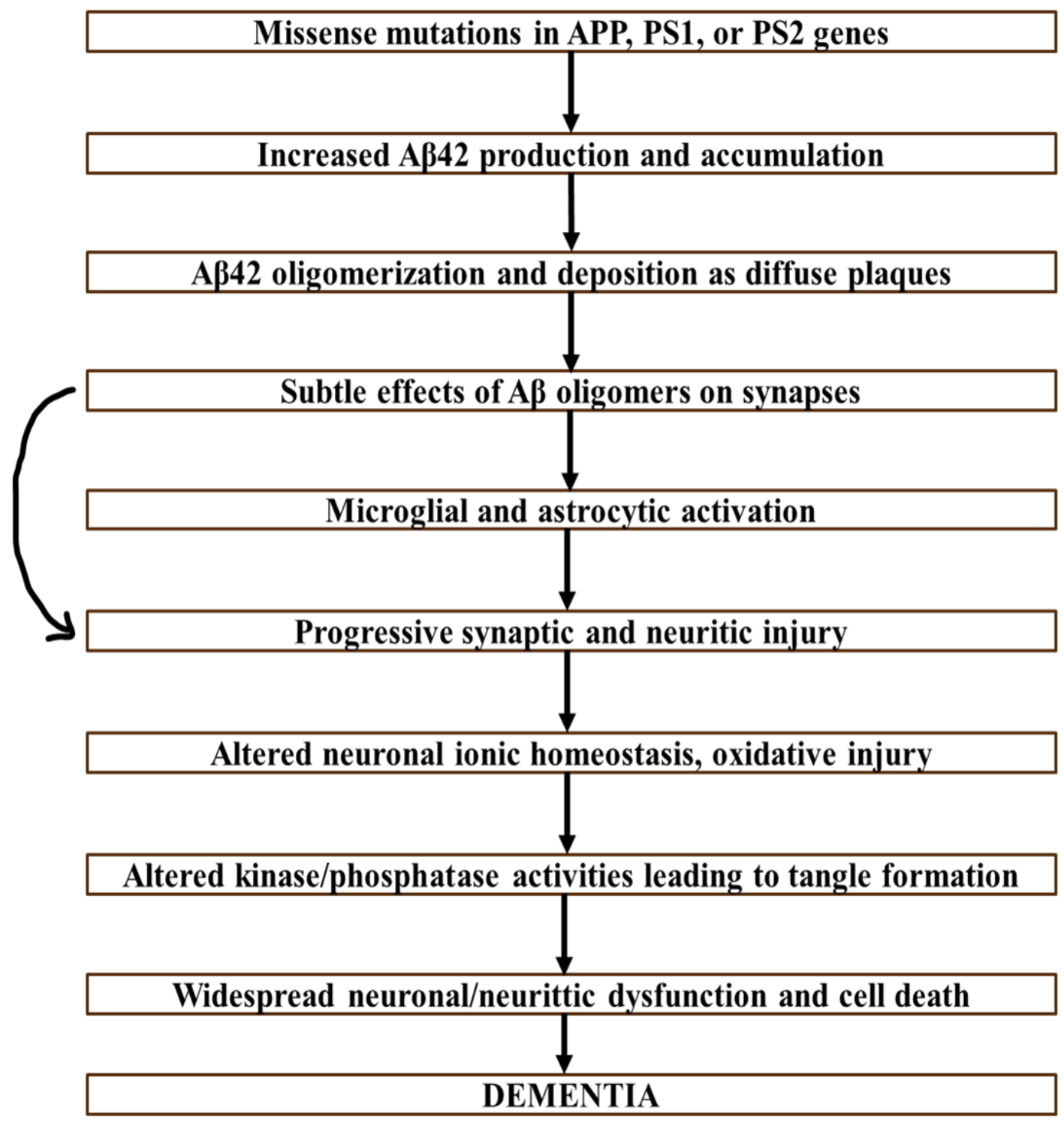

Figure 1: The sequence of pathogenic events as outlined by the amyloid cascade hypothesis. The curved black arrow indicates that beta-amyloid oligomers may cause damage to the synapses and neurons directly, in addition to activating microglia and astrocytes [adapted from 38]. 


\subsection{Clinical Expression}

In accordance with the cognitive impairment observed in patients, $\mathrm{AD}$ is thought to occur in three stages- preclinical $\mathrm{AD}, \mathrm{MCI}$ due to $\mathrm{AD}$ and dementia due to $\mathrm{AD}(3)$. The differences between typical age-related cognitive changes and changes induced by Alzheimer's dementia can be subtle in the pre-dementia stage. The physical symptoms associated with $\mathrm{AD}$ include difficulty in planning or solving problems, challenges in completing familiar tasks, difficulty in interpreting visual cues and spatial relationships, decreased or poor judgment, misplacing things and changes in mood and personality among others (2). The symptoms observed in $\mathrm{AD}$ are often progressive and worsen with time.

The changes in preclinical $\mathrm{AD}$, the first stage, are still under investigation and can often be subtle, occurring ten to fifteen years prior to the appearance of observable symptoms in AD (1). While studies have observed measurable changes in brain, cerebrospinal fluid (CSF) and blood-based biomarkers that are indicative of the earliest signs of $\mathrm{AD}$, additional research is required to finetune the accuracy of these tools before they become available for use in clinical settings (43). Preclinical AD is often marked by changes in mood and personality of individuals causing them to become confused, depressed or anxious in places where they are out of their comfort zones. 
Mild cognitive impairment (MCI) due to AD is the subsequent stage in AD. People suffering from MCI show biomarker evidence of AD related brain changes such as elevated levels of beta-amyloid deposition in their brains. Consequently, they exhibit greater cognitive decline than expected for their age, but it does not interfere with their daily activities (43). In the MCI stage, patients are able to work independently in most areas in their life, but they likely require some assistance with certain activities to maximize their independence and to remain safe. An estimate of $15 \%$ of all patients suffering from MCI convert to clinical AD, the final stage of illness (44).

In the clinically manifested stage of $\mathrm{AD}$ illness i.e. dementia due to Alzheimer's, the effects of $\mathrm{AD}$ on the physical health of a patient become especially apparent. Noticeable changes in memory, thinking and behavioral profile are characteristic of patients suffering from dementia due to $\mathrm{AD}$ which impair their ability to function in daily life. Because of the damages to the areas of brain involved in motor movements, coordination and memory; by the clinical AD stage patients become bed-bound with limited ability to verbally communicate. These changes in patients' physical health and personality are first observed by family members or primary healthcare members, which prompts them to seek medical attention. 


\subsection{Current State of Diagnosis}

Since dementia due to Alzheimer's is thought to be caused by multiple factors, there is no single test to diagnose it. Physicians along with the help from specialists such as neurologists, geriatricians and geriatric psychiatrists use several criteria before they confer a diagnosis of AD. These criteria include obtaining a medical and family history from the patients, conducting cognitive tests, neurologic and physical examinations, using brain imaging tools to check for deposition of high levels of beta-amyloid and asking feedback from family members regarding changes in thinking skills and behavior (45).

For diagnosis of $\mathrm{AD}$, the National Institute of Neurological and Communicative Disorders and Strokes and the Alzheimer's disease and Related Diseases Association (NINCS-ADRDA) criteria has been used as the gold standard. As per the NINCS-ADRDA criteria, for a patient to receive a probable AD diagnosis, they must satisfy the following conditions: onset and worsening of characteristic $\mathrm{AD}$ symptoms for a period of many months or a few years, memory deficits including inability to recall new learned information and forgetting names or appointments but remembering them later, and the onset of nonamnestic symptoms such as decline in language abilities, loss of coordination and executive functions among others (46). 
Various neuropsychological examinations are incorporated in clinical diagnosis of $\mathrm{AD}$ to supplement clinical interviews and brain imaging tests. Mini-Mental State Examination (MMSE) (45), Clinical Dementia Rating (CDR) (47), Montreal Cognitive Assessment (MoCA) (48) and Functional Assessment Questionnaire (FAQ) (49) are the most common neuropsychological examinations being conducted in AD diagnosis. FDG PET and MRI based brain scans of AD patients are often used to complement clinical diagnosis by clinicians.

Currently, it is well accepted that patients suffering from MCI are at higher risk of progression to $\mathrm{AD}$ dementia when compared to cognitively normal adults. Studies have shown that approximately $20 \%-40 \%$ of amnestic MCI patients convert to AD dementia (44). Therefore, early intervention for MCI patients prone to conversion to $\mathrm{AD}$ has been the focus of healthcare practices around the globe. Physicians recommend regular interviews and neuropsychological assessments of individuals suffering from MCI for early detection and management of $\mathrm{AD}(48)$.

To complement the traditional clinical diagnosis of $\mathrm{AD}$, use of radiofluorinated $\left[{ }^{18} \mathrm{~F}\right]$ FDG PET has been commonly accepted (40). FDG PET is used to measure regional glucose metabolism by estimating cerebral 
metabolic rate of glucose (CMRglc) (50). Several FDG PET-based automated biomarkers have been established which have helped to obtain highly objective and sensitive clinical diagnosis in patients with suspected neurodegenerative disorders (8). These biomarkers and classifiers have especially helped in separating patients suffering from $\mathrm{AD}$ dementia from other forms of dementia which mimics AD symptoms (9).

Due to the lack of a standardized scanning protocol, the current method of examining FDG PET scans of patients by nuclear medicine physicians has not been standardized across globe; let alone individual countries like Canada or United States. This induces inter-physician variability as the reading techniques vary vastly from one physician to another. This variability among physicians is also caused by factors such as their training obtained during residency, personal preferences in methodologies and the amount of expertise and training they have in the same field (10). Also, the differences in PET cameras, imaging protocols, and image reconstruction algorithms, etc, induces variability in PET images of patients. Hence the variabilities in examining FDG PET scans pose a challenge in differential diagnosis of AD from other forms of dementias, and in early diagnosis. 


\subsection{Treatment of Alzheimer's Disease}

The life expectancy of individuals who have been diagnosed with AD is approximately 7-10 years. Currently, none of the pharmacologic treatments or medications available for $\mathrm{AD}$ slow the progression of $\mathrm{AD}$ or stop the loss of neurons that make the disease fatal. The process of developing effective treatments for $\mathrm{AD}$ is limited by factors such as the slow pace of recruitment of patients into clinical studies and large time periods needed to observe whether investigational drugs affect disease progression (51). Design of pharmacologic drugs for $\mathrm{AD}$ treatment is also hindered by the gap in knowledge about the precise molecular changes and biological processes responsible for $\mathrm{AD}$ pathology.

The treatment options available for $\mathrm{AD}$ patients consist of administration of cholinesterase inhibitors such as donepezil, memantine or galantamine. As of 2019, six drugs have been approved by the U.S. Food and Drug Administration (FDA) for treatment of Alzheimer's symptoms- rivastigmine, galantamine, donepezil, memantine, memantine combined with donepezil, and tacrine. In $40-58 \%$ of responder cases, these drugs have been found to be successful in providing symptomatic relief in AD patients (52). 
Acetylcholinesterase inhibitors such as donepezil work by preventing depletion of acetylcholine within the synaptic cleft. This results in an increase in cerebral acetylcholine levels which promote cholinergic neurotransmission within the brain (53). In addition, other drugs are currently being developed which could serve as potential treatment options in the future. P7C3, a derivative of the antihistamine drug dimebon, has been shown to have neuroprotective properties via binding to the serotonin receptors in brain (54). Other potential treatments include the administration of $\alpha$-melanocyte stimulating hormone and metal-protein attenuating compounds (MPACs) such as PBT2 (54).

Non pharmacologic therapies have been studied in patients with $A D$ dementia with the goal of maintaining or improving cognitive function in these patients (54). These therapies are targeted at reducing behavioral symptoms among AD patients such as depression, apathy, sleep disturbances, agitation and aggression. Repetitive transcranial magnetic stimulation (rTMS) combined with cognitive training has been suggested as safe and effective modality for treatment of $\mathrm{AD}$ (55). Studies have shown that exercise has a positive effect on overall cognitive function, and hence it can help Alzheimer's patients by slowing the rate of cognitive decline in these patients (34). Similarly cognitive 
stimulation, ranging from object categorization exercises to reality orientation exercises, has been shown to have beneficial effects in cognitive functions of people with Alzheimer's dementia $(54,55)$.

\subsection{Overview of PET Imaging}

PET is an analytical nuclear imaging modality developed to use radioisotopes as molecular probes to image and measure biochemical processes in vivo (56). PET imaging involves administration of compounds labeled with positron emitting radioisotopes, called radiotracers, into the subjects to provide diagnostic information on various physiological processes. Traditionally, PET imaging has been used to measure glucose metabolism, tissue perfusion, the activity of specific receptors in the brain and gene expression.

The positron emitting radiotracers used in PET imaging have a proton rich nuclei which attempts to stabilize itself by getting rid of the excess protons. In this process, the positron emitting radiotracer stabilizes by decay of a proton into a positron, neutron and a neutrino. Schematically the process can be represented by:

${ }_{\mathrm{m}} \mathrm{X}^{\mathrm{n}}$----------------------------------->${ }_{\mathrm{m}-1} \mathrm{Y}^{\mathrm{n}}+\beta^{+}+\mathrm{v}$ 
There occurs a transmutation of elements (i.e. from element $\mathrm{X}$ to $\mathrm{Y}$ ) with the resulting nucleus having same mass number, but the atomic number reduced by 1 . The positron produced in this process has a very short life time and combines with an electron resulting in production of two photons through mass energy conversion as shown in the equation below:

$$
{ }_{+1} \beta^{0}+{ }_{-1} \mathrm{e}^{0} \text {------------------> } 20 \gamma^{0}
$$

The two photons produced as a result of this reaction have equal energy of about $511 \mathrm{keV}$ and have approximately $180^{\circ}$ degree separation (antiparallel) between them. These antiparallel photons are detected by gamma ray detectors creating a line of response (LOR). The opposite PET detectors register the arrival of these antiparallel photons to acquire these coincidence events. Using these coincidence events of annihilation photons, a map of the distribution of radiotracers is determined to approximate the regions with higher tracer activity (57).

Commonly used PET radiotracers are labelled with radionuclides such as ${ }^{11} \mathrm{C},{ }^{13} \mathrm{~N},{ }^{15} \mathrm{O}$ and ${ }^{18} \mathrm{~F}$. Clinically the most commonly used radiotracer in PET imaging is ${ }^{18} \mathrm{~F}$ labelled fluorodeoxyglucose $\left({ }^{18} \mathrm{~F}-\mathrm{FDG}\right)$, a glucose analog labelled with isotope fluorine-18 (58). ${ }^{18} \mathrm{~F}$-FDG tracer localizes in cells with high rate of glucose utilization; hence it has diagnostic utility in oncology and 
neurological disorders. Increased FDG uptake in a region reflects increased metabolism (or 'hypermetabolism') in that brain region, while decreased FDG uptake reflects decreased metabolism (or 'hypometabolism') in a brain region. ${ }^{18} \mathrm{~F}-\mathrm{FDG}$ is useful in diagnosis of neurological disorders due to difference in rate of cerebral glucose utilization in patients as compared to normal controls. Similarly, as tumor tissues consume more glucose than surrounding tissues in cancer patients, ${ }^{18} \mathrm{~F}$-FDG uptake serves as an indicator of proliferation of tumor cells and degree of malignancy (58).

\subsection{Factors influencing FDG uptake in AD}

Skoloff's radiographic method for measuring cerebral metabolic rate of glucose (CMRglc) using $\left[{ }^{14-} \mathrm{C}\right]$ deoxyglucose laid the foundation of modern FDG PET imaging. FDG PET based brain imaging has been established as the benchmark for $\mathrm{AD}$ neuroimaging diagnosis, however subjective impression of FDG PET readings limits its use in clinical practice (10). Standardized automated analysis of FDG PET is increasingly used to provide an objective and sensitive support to clinical diagnosis in patients with suspected major neurodegenerative disorders like AD, dementia with lewy body (DLB) and frontotemporal dementia (FTD) (59). 
Glucose utilization has been shown to correlate with neuronal activity (50), hence activity dependent accumulation of radiofluorinated $\left[{ }^{18} \mathrm{~F}\right]$ FDG brain uptake is viewed as a proxy for neuronal activity (58). However adult rat model studies have shown that activation of astrocytic glutamate transport triggers widespread glucose uptake in the rodent brain (60). This calls for revaluation of the contribution of glial cells to neuroenergetics. Even though stimulation of astrocytic glutamate uptake may increase glucose utilization, brain glucose metabolism is still indicative of synaptic activity (61).

Studies have shown decreased activities of key enzymes involved in energy metabolism such as cytochrome c oxidase and $\alpha$-ketoglutarate dehydrogenase complex, in brains of $\mathrm{AD}$ patients (62). In addition, altered proteolytic processing of APP protein has been associated with impaired energy metabolism. Transgenic APP mice studies have established that transgenic APP mice exhibit age-dependent reduction in glucose metabolism in brain regions associated with cognitive processes (63). These findings suggest that perturbed glucose metabolism play a role in $\mathrm{AD}$ pathogenesis.

There has been increased research interest in the differential contribution of neurofibrillary tangles and beta-amyloid burden on the degree of neurodegeneration measured by glucose metabolism in $\mathrm{AD}$ patients. The 
accumulation of neurofibrillary tau tangles (measured with $\left.\left[{ }^{18} \mathrm{~F}\right] \mathrm{AV}-1451\right)$ ) has been associated with hypometabolism in AD patients (64). Studies have found no correlation between mean amyloid deviation (measured with $\left[{ }^{11} \mathrm{C}\right] \mathrm{PIB}$ ) and mean FDG deviation suggesting that there is no distinct regional relationship between $A \beta$ deposition and neurodegeneration (37). A recent study using transgenic APP/PS1 mice model suggested a synergistic effect of A $\beta$ mediated tau-propagation and neuronal loss (65).

Across brain regions in $\mathrm{AD}$ patients, an interactive effect of beta-amyloid burden and tau deposition on glucose metabolism was observed most prominently in the parietal lobe (37), which supports the hypothesis of synergistic effect of $A \beta$ induced tau propagation resulting in $A D$ related hypometabolism. However, factors that may increase the brain glucose metabolism in AD have not been thoroughly investigated.

\subsection{Alzheimer's Disease Neuroimaging Initiative (ADNI)}

The Alzheimer's Disease Neuroimaging Initiative (ADNI) is a longitudinal multicenter study. The main aim of ADNI is to develop clinical, imaging and genetic biomarkers for the early diagnosis of $\mathrm{AD}$ (http://adni.loni.usc.edu/about/). ADNI is a combined effort of multiple research centres across North America which have collected data from thousands of AD, 
MCI and normal subjects across the region with the purpose of tracking AD conversion and progression.

The main goals of ADNI study are:

i) To develop biomarkers which would aid in detection of $\mathrm{AD}$ at the earliest possible stage (pre-dementia) and help in tracking disease progression.

ii) To design new diagnostic methods for $\mathrm{AD}$ diagnosis which would make intervention possible at the earliest possible stages of disease; thereby supporting most effective advances in $\mathrm{AD}$ intervention, prevention, and treatment.

iii) To regularly administer ADNI's data-access policy with an aim to provide all data without embargo to scientists around the world.

The ADNI project was launched in 2004 and is funded by the National Institute on Aging (NIA) and the National Institute of Biomedical Imaging and Bioengineering (NIBIB) of the National Institutes of Health (NIH), the Food and Drug Administration (FDA), several private pharmaceutical companies , and foundations (Alzheimer's Association, Institute for Study of Aging) in conjunction with the NIH Foundation (66). It was launched as a $\$ 60$ million, 5year public private partnership with the primary goal to investigate the 
combined utility of positron emission tomography (PET), magnetic resonance imaging (MRI), other genetic and biological markers and neuropsychological assessments to measure the progression of mild cognitive impairment (MCI) and $\mathrm{AD}(67)$.

The depth of imaging, genetics and biological marker data available on ADNI has made several valuable discoveries possible. It has provided a platform to detect $\mathrm{AD}$ at the earliest possible stage (pre-dementia) by development of novel ways of diagnosing early $\mathrm{AD}$ and tracking disease progression through biomarkers such as CSF-tau and amyloid beta levels (66). Development of specific biomarkers sensitive to changes in early $\mathrm{AD}$ progression will aid researchers and clinicians in development of new therapeutic treatments for AD (67). These biomarkers will also lessen the time and cost of clinical trials by providing an effective way to monitor the effectiveness of treatments (68).

Utilizing the ADNI database, a number of studies have established quantitative biomarkers that may aid in $\mathrm{AD}$ diagnosis (12-15). Various automated diagnostic tools have been developed for objective diagnosis of $\mathrm{AD}$ patients which are sensitive to early cognitive changes in $\mathrm{AD}$ progression. $\mathrm{A}$ comparative study of diagnostic accuracy between a quantitative software-aided 
approach and visual analysis found that the sensitivity, specificity and accuracy of software-aided approach in classifying dementia patients from normal controls was higher than traditional visual analysis (12).

A comparative study on predictive utility reported significant increase in accuracy in predicting conversion to AD when MR imaging, FDG PET and CSF data are combined with routine clinical tests as compared to clinical tests alone (13). The use of multiparametric PET obtained by combining quantitative functional FDG PET and pathological amyloid- $\beta$ PET, along with cerebrospinal fluid (CSF) biomarkers and clinical neuropsychological assessments have potential clinical utility in prediction of $\mathrm{AD}$ progression (14). However, the presence of CSF-tau is not specific to AD dementia as high CSF-tau concentration can be observed in one patient with $\mathrm{AD}$ dementia and also in patient with some other form of dementia.

\subsection{Graph Theory}

Graph theory is the branch of mathematics that deals with the study of graphs, which are mathematical structures used to model pairwise relationships between objects (69). Graphs are comprised of nodes, representing an object of substantive interest, which are connected by edges signifying the existence of a relationship between the two objects. Graph theory has traditionally been used 
to facilitate the understanding of relational structure represented by complex graphs; and to aid in better comprehension of relational dynamics by simplification and reorganization of a complex graph (70).

Swiss mathematician Leonhard Euler introduced the basic idea of graphs in the $18^{\text {th }}$ century to solve the Königsberg bridge problem. Since then, graph theory has been used in a diverse range of problems to model the relations and process in physical, biological (71), social (72) and information systems. Graph theory has been famously used in modern day social network analysis to model social interactions between individual users on social networking platforms such as Facebook, Instagram, etc (72). There has been increased research interest to evaluate the potential utility of graph theory in modelling of realworld systems. For instance, it is being used in computer science to represent networks of communication and data organization, it is being used in biology to track disease propagation (73), and it is being used in condensed matter physics to study the three-dimensional structure of complicated simulated atomic structures.

Global and local graph theoretic metrics are evaluated to model and analyze the complex relational structure and dynamics of a graph. Global metrics such as Characteristic Path Length (L) and Clustering Coefficient (C) 
are commonly used to measure efficiency and resilience of a graph respectively. A 'path' in a graph is defined as the sequence of distinct connected nodes in a network. The 'geodesic' distance $d(i, j)$ between nodes $i$ and $j$ is defined as the length of the shortest path between these two nodes, which is measured by the number of lines traversed to reach from $\mathrm{i}$ to $\mathrm{j}$. The average distance for a node $\mathrm{i}$ $[\bar{d}(\mathrm{i})]$ to all other nodes in the network is defined as $\bar{d}(\mathrm{i})=1 /(\mathrm{n}-1) \sum_{j=1}^{n} d(i, j)$. $\mathrm{L}$ is the average of these shortest path lengths of all the nodes in the network, or $\mathrm{L}=1 / \mathrm{n} \sum_{i=1}^{n} \bar{d}(\mathrm{i})(74)$.

The $\mathrm{C}$ of a graph was introduced by Watts and Strogatz in context of social network analysis. $\mathrm{C}$ of a graph measures the cliquishness of a typical graph neighborhood and is a measure of the degree to which nodes in a graph tend to cluster together (75). Given three nodes $\mathrm{p}, \mathrm{q}$ and $\mathrm{r}$ in a graph with mutual relations between $\mathrm{p}$ and $\mathrm{q}$ and $\mathrm{p}$ and $\mathrm{r}, \mathrm{C}$ represents the likeness that $\mathrm{q}$ and $\mathrm{r}$ are also related. $\mathrm{C}$ is the fraction of nearest neighbors of a node that are in turn nearest neighbors of each other (76). $\mathrm{C}$ of a graph has become a frequently used tool for analyzing graphs as evidence suggests that in most real-world networks, nodes tend to create tightly knit clusters characterized by relatively high density of connections or ties between the clusters $(75,77,78)$. 
Betweenness Centrality, a local graph theoretic measure, is the measure of 'hubness' of a node and is represented by the fraction of shortest paths passing through the node (79). BC measures define "centrality in terms of the frequency with which a node falls on the shortest path between others; and thus $\mathrm{BC}$ has a potential for control of communication in a network" (80). BC algorithm is designed to rank the nodes according to their position in the network and is interpreted as the prominence of nodes in the information flow structure of the network. For information to efficiently transfer within a network, it will pass more frequently through the nodes with the maximum number of shortest paths passing through them (79).

\subsection{Application of Graph Theory in AD}

Amyloid- $\beta$ plaques and tau related neurofibrillary tangles, the hallmarks of AD pathology, have been associated with local synaptic disruptions $(81,82)$. Studies have also established that during disease progression in $\mathrm{AD}$, cortical atrophy spreads in a systematic order from subcortical structures like the hippocampus into associative cortical areas and finally reaches into primary sensory areas $(83,84)$. These findings advocate that $\mathrm{AD}$ is a dysconnectivity disease and certain cortical areas are more vulnerable for AD pathology, which in turn could be used to determine how $\mathrm{AD}$ propagates across different paths in 
a network. This also leads to the conclusion that if $\mathrm{AD}$ is indeed a dysconnectivity disease, $\mathrm{AD}$ progression could be captured with a graph theory based network approach as the structural elements of the brain form complex networks at various spatial scales which in turn give rise to functional dynamics (85).

There has been increased research interest to investigate the structural and functional properties of abnormal networks associated with AD. Graph theory models interrelationships (denoted via edges on a graph) between different brain regions (denoted via nodes on a graph) and estimates the properties of brain networks using different graph theoretic metrics (86). Several studies have shown that modelling of the brain via graph theory is critical in the understanding of underlying brain networks in psychiatric and neurological disorders $(87,88)$. Graph theory has been efficiently used to characterize different aspects of brain networks in $\mathrm{AD}$ patients by evaluating measures of integration and segregation via graph theoretic metrics.

A recent study showed that graph measures generated for brain networks on the basis of resting-state (RS) functional magnetic resonance imaging (fMRI) scans were able to accurately classify AD patients from healthy controls with high accuracy (22). In a study investigating the connectivity patterns of the 
default mode (DMN), frontoparietal, executive and salience networks, it was found that $\mathrm{AD}$ was associated with opposing connectivity effects in DMN (decreased) and frontal networks (increased) (89). The study also found that the mean executive network connectivity in $\mathrm{AD}$ patients was highly correlated with frontal-executive and language neuropsychological scores. These results are evidence of alteration of large-scale functional brain networks in $\mathrm{AD}$ which extend well beyond the DMN.

$\mathrm{AD}$ disrupts the whole-brain topological organization of the functional connectome which provides novel insights into the pathophysiological mechanism of $\mathrm{AD}$ (90). Along with the disruption in the topological architecture of whole brain connectivity, several research groups have reported redistribution of hubs in $\mathrm{AD}$ connectome $(91,92)$. Studies have suggested that there is loss of hubs in $\mathrm{AD}$ network as compared to normal controls (93). The loss of hubs in $\mathrm{AD}$ might be explained by atrophy of particular areas in $\mathrm{AD}$ as the areas like temporal region, insula, cingulate are known to be lesioned in $\mathrm{AD}$ (94). 


\section{Chapter 3: Experimental Approach}

In this chapter, details on experimental methodology used in the study are provided. Data used in the preparation of this article were obtained from the Alzheimer's Disease Neuroimaging Initiative (ADNI) database (http://adni.loni.usc.edu/).

\subsection{Subjects}

The ADNI database comprised of 1000 subjects including 200 Alzheimer's (AD), 200 Normal Controls (NL) and 600 mild cognitive impairment subjects (MCI) who have been recruited from across more than 50 sites in the US and Canada (68). Longitudinal imaging data including structural MRI and FDG PET scans, along with neuropsychological and clinical assessments were collected at baseline, and at follow-up visits occurring at sixto twelve-month intervals. For each study site, approval from Institutional Review Board was obtained prior to start of study. Written informed consent was obtained from all study participants, or their authorized representatives.

Diagnosis of $\mathrm{AD}$ was conferred as per the criteria established by the National Institute of Neurological and Communicative Diseases and StrokeAlzheimer's Disease and Related Disorders Association criteria for probable AD (95). Similarly, diagnosis for amnestic MCI was conferred if the patient 
reported memory complaints and presented with objective memory deficits, intact functional activities, and had Clinical Dementia Rating (96) (CDR) Scale global score of 0.5 , and a MMSE (97) score of 24 or more.

Based on availability of FDG PET and MRI scans at baseline, 116 AD patients were selected from the ADNI cohort. FDG PET and MRI scans of each of these subjects were visually inspected to assure quality. Based on availability of whole brain scans (i.e. checking whether entire cerebellum is included in FDG-PET and MRI scans), 28 AD subjects were excluded which had partial scans of cerebellar regions. Hence, finally we selected 88 AD patients using this selection protocol for $\mathrm{AD}$ network analysis and biomarker derivation. Of the 200 normal controls available in ADNI database, 154 had FDG PET and MRI scans available. Of the 154 normal controls, based on image quality assurance and follow up period of 36 months, 127 normal controls were selected. 88 agesex matched normal controls (NL1) were selected from this cohort for comparative network analysis. Demographic details of subjects included in the study from ADNI cohort are mentioned in Table1.

For an additional longitudinal study, MCI subjects were further categorized into two categories: stable MCI (sMCI), subjects who remained MCI after a follow up greater than three years and Prodromal AD (PAD), MCI 
subjects who developed Alzheimer's later during follow up period. Of the 445 MCI subjects for which FDG PET and MRI scans were available, 54 converted to $\mathrm{AD}$ and 186 subjects remained stable MCI (sMCI). 39 PAD subjects $(n=39)$ were selected for whose scans were available at three time points (conversion year, 1 year prior to conversion and 2 years prior to conversion). Of the 186 sMCI subjects, 67 subjects had FDG PET and MRI scans available at three consecutive time points. Of these, 39 sMCI subjects to match the sex and age of 39 PAD patients. Similarly, 39 normal controls (NL2) were selected among whose PET and MRI images are available at baseline and two-year follow-up. It should be noted that NL2 is a subgroup of NL1.

We carefully selected the sub-samples of control groups (sMCI, NL1, NL2) to match the sample size of the active groups (AD and PAD) because the graphs were constructed from group-wise correlation matrices; and the significant group difference of which was tested via random permutation procedure. Subject selection from ADNI database is shown in Figure 2. 


\begin{tabular}{|l|l|l|l|l|l|l|l|}
\hline & AD & NL1 & $\begin{array}{l}\text { t-test } \\
\text { (AD vs } \\
\text { NL1) }\end{array}$ & PAD & $\begin{array}{l}\text { sMCI(matched } \\
\text { for PAD) }\end{array}$ & NL2 & $\begin{array}{l}\text { ANOVA } \\
\text { (AD vs } \\
\text { NL1 vs } \\
\text { sMCI) }\end{array}$ \\
\hline $\begin{array}{l}\text { Number } \\
\text { of } \\
\text { Patients }\end{array}$ & 88 & 88 & & 39 & 39 & 39 \\
\hline $\begin{array}{l}\text { Age } \\
\text { Mean } \pm \text { SD } \\
\text { max })\end{array}$ & $\begin{array}{l}75.23 \pm 7.29 \\
(63-92)\end{array}$ & $\begin{array}{l}74.66 \pm 8.32 \\
(56-90)\end{array}$ & $\mathrm{p}=0.6247$ & $\begin{array}{l}74.52 \pm 7.47 \\
(60-87)\end{array}$ & $\begin{array}{l}73.91 \pm 8.16 \\
(59-90)\end{array}$ & $\begin{array}{l}72.96 \pm 8.83 \\
(63-88)\end{array}$ & $\mathrm{p}=0.4792$ \\
\hline $\begin{array}{l}\text { Sex(M:F) } \\
55: 33\end{array}$ & $54: 34$ & $\mathrm{p}=0.8775$ & $25: 14$ & $24: 15$ & $23: 16$ & $\mathrm{p}=0.3671$ \\
\hline MMSE & $\begin{array}{l}23.6 \pm 2.2 \\
(20-26)\end{array}$ & $\begin{array}{l}28.8 \pm 1.1 \\
(26-30)\end{array}$ & $\mathrm{p}=0.5437$ & $\begin{array}{l}26.7 \pm 2.3 \\
(24-30)\end{array}$ & $\begin{array}{l}27.9 \pm 1.6 \\
(24-30)\end{array}$ & $\begin{array}{l}28.9 \pm 1.0 \\
(26-30)\end{array}$ & $\mathrm{p}=0.2851$ \\
\hline
\end{tabular}

TABLE 1

Table1: Demographic data for patients. 


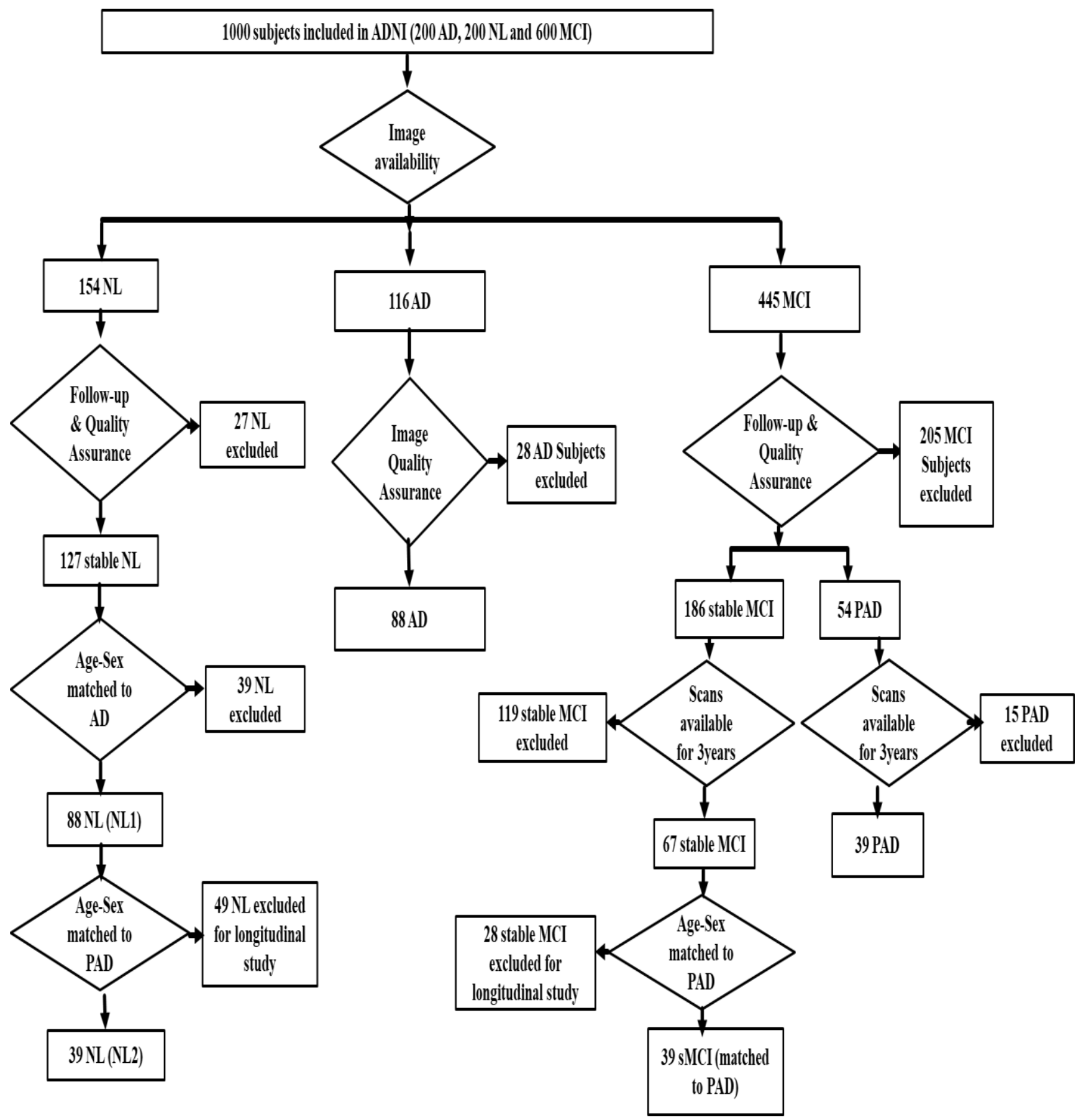

Figure 2: Subject Selection Protocol. ADNI database comprised of 1000 subjects including 200 Alzheimer's patients (AD), 200 Normal Controls (NL) and 600 mild cognitive impairment subjects (MCI). Based on availability of 
PET and MRI scans, 116 AD patients were selected. 28 AD patients were excluded after image quality assurance such as inclusion of whole cerebellum in PET scans. Finally, 88 AD patients were selected for biomarker derivation and network analysis. Follow up duration was neglected for $\mathrm{AD}$ as dementia was already present at the time of screening in the subjects. Out of the $154 \mathrm{NL}$ subjects with available PET and MRI scans, 127 remained NL after a follow up of three years. 88 age-sex matched NL subjects were selected from the 127 stable NL subjects. Of the 445 MCI subjects for which PET and MRI scans were available, based on a follow up period of atleast three years, 186 remained stable MCI and 54 converted to AD (PAD). 39 PAD subjects had scans available at three time points (conversion year, 1 year prior to conversion and 2 years prior to conversion). For longitudinal FDG-SUR analysis, 39 age-sex matched stable MCI and NL subjects were selected which had scans available at three time points. 


\subsection{Image Acquisition}

FDG PET images for the study were downloaded from publicly available online ADNI Laboratory of Neuroimaging (LONI) database. Pre-processed images where frames had been co-registered to one another and averaged, followed by standardization were acquired from the LONI database. For FDG PET images, six five-minute frames are acquired 30 to 60 minutes post injection, and each frame is realigned to the first frame.

The realigned images are averaged, and the averaged images are then reoriented to 160x160x96 standard voxel image grid. These images are then filtered with a scanner specific function resulting in images of a uniform isotropic resolution of $8 \mathrm{~mm} \mathrm{FWHM,} \mathrm{the} \mathrm{approximate} \mathrm{resolution} \mathrm{of} \mathrm{the} \mathrm{lowest}$ resolution scanners used in ADNI. The detailed procedure followed in preprocessing of FDG PET images in ADNI can be found at: http://adni.loni.usc.edu/methods/pet-analysis-method/pet-analysis/\#pet-preprocessing-container.

Structural MRI images used in pre-processing of FDG PET images were also acquired from LONI database for each scan. The MRI scans were obtained from a 3T T1-weighted images, which were typically 240 x 256 x 176 voxels with a voxel size of $2 \mathrm{~mm} \times 2 \mathrm{~mm} \times 2 \mathrm{~mm}$ (98). To correct the MRI images for 
the geometric distortion induced by gradient non-linearity, MR image corrections such as GradWarp were applied (95). These corrections also correct for distortions induced by bias field due to the absence of uniformity in the radio frequency receiver coils (99). The detailed procedure followed by ADNI in MRI image acquisition can be found at: http://adni.loni.usc.edu/methods/mri$\underline{\text { tool/mri-analysis/ }}$.

\subsection{Image Preprocessing}

FDG PET images were preprocessed using standard preprocessing pipeline using statistical parametric mapping 12 (SPM 12) software (www.fil.ion.ucl.ac.uk/spm/). FDG PET images were coregistered to structural MRI images using rigid body transformation in SPM12. CAT12 was used to segment structural MRI images and generate forward deformation fields from MRI used in normalization (http://www.neuro.uni-jena.de/cat/). The segmentation routine by CAT12 segments and spatially normalises the MRI segments all in the same model.

FDG PET images were normalized to standard MNI (Montreal Neuroimaging Institute) space using forward deformation fields. The normalized images were subsequently smoothed using an $8 \mathrm{~mm} \times 8 \mathrm{~mm} \times 8 \mathrm{~mm}$ Gaussian filter. Smoothing is used to suppress noise artefacts arising due to 
residual differences in functional and gyral anatomy during inter-subject averaging.

\subsection{FDG PET based AD Classifier (FAC)}

The preprocessed FDG PET images were proportionally scaled to whole brain and WM mean for signal intensity normalization. We derived $\mathrm{AD}$ classifier using whole brain mean (as used in conventional studies), and WM mean as well. The metabolic topography and performance of classifiers based on both reference regions was evaluated and compared.

The brain mask was produced by combining the grey matter and white matter masks from each subjects' segmented structural MRIs and used as an inclusive mask, as previously described (8). For each voxel within the brain mask, we used a linear regression model of least squares which can be represented by equation 3 :

$$
\mathrm{Y}=\beta * \mathrm{X}+\mathrm{C}
$$

In this equation, $\mathrm{X}$ is the dummy variable used for group classification, $\mathrm{Y}$ is subject's observed image, $\beta$ is the slope of regression model and $\mathrm{C}$ is the constant. Using this regression model, $\beta$ map was constructed. The dot product between the reconstructed (i.e., FAC) and each individual's proportionally scaled images within the brain mask is defined as a subject score, which 
differentiated the two subject groups (AD vs NL) (8). We used 10-fold cross validation to test the predictive accuracy of classifier model for an independent dataset, and to check for problems like overfitting or selection bias.

The AAL atlas was masked with the whole brain mask that was described above. Pons were added to the original AAL atlas as described elsewhere (25). The mean value of each of 118 regions-of-interest were extracted from FDG PET images of all subjects, then proportionally scaled to the mean value of whole brain WM mean. Using the same regression model used in voxel-based analysis, slope coefficient $\beta$ is calculated for all ROIs. Based on their $\beta$ values $(\beta>0$ or $\beta<0)$ and Bonferroni corrected $p$-values for the model $[\mathrm{p}<$ (0.05/number of ROIs)], regions are classified as hypermetabolic or hypometabolic respectively. Regions with $\mathrm{p}>0.05 /$ number of ROIs were classified as non-significant (NS). To test reproducibility and interchangeability of voxel-based vs. ROI-based FAC, topographical similarity was evaluated by Pearson's correlation. For this, the mean of $\beta$ values from each ROI was extracted from the voxel-based $\beta$ map. 


\subsection{AD Network Analysis Using Graph Theory}

Normalized metabolic activity with respect to WM mean in all ROIs is used to generate a region * region correlation matrix for $\mathrm{AD}$ group and $\mathrm{NL}$ group, separately. The absolute r-values were ranked and undirected unweighted adjacency matrices were generated at varying cost thresholds (1$50 \%)(100)$. Cost threshold is a measure of network density of the graph and is defined as the proportion of actual edges to the maximum number of possible edges in a graph. For instance, a graph thresholded at network density of $30 \%$ corresponds to an undirected adjacency matrix generated by setting the top 30\% elements in absolute correlation matrix to 1 , and the rest of the elements including diagonal elements are set to 0 . Minimum network density is defined as the minimum cost at which all nodes become fully connected in brain networks of both groups and was found to be at 16\% (101).

To compare the overall network efficiency between AD and NL1, we computed Characteristic Path Length (L) and Clustering Coefficient (C) (75) at varying cost thresholds of $16-25 \% . \mathrm{L}$ is a measure of the average shortest path length between all pairs of nodes in a network and represents the efficiency of interaction of different nodes in a network. $\mathrm{C}$ is the fraction of node's 
neighbours that are in turn nearest neighbours of each other and represents the resiliency of the network.

To compare the relative nodal importance within the constructed graphs of $\mathrm{AD}$ vs. NL1, we computed Betweenness Centrality (BC) as a measure of hubness of each region in the network for the cost threshold range 16-25\% (22). $\mathrm{BC}$ is the fraction of all shortest paths in the network that contain a given node and approximates the role of a region in information transfer through the network (102). Nodes with high values of BC participate in a large number of shortest paths, and thus play a significant role in information transfer acting as 'hubs' in the network.

In order to test for statistical significance of the group differences between $\mathrm{AD}$ and $\mathrm{NL}$ groups in $\mathrm{C}, \mathrm{L}$ and $\mathrm{BC}$, a non-parametric permutation test with 1000 iterations was used (91). The data from subjects from both groups is merged into a combined subject pool. For each iteration, subjects are randomly reassigned into one of the two groups; so that each randomized groups has an equal number of subjects as the original two subject groups. Then correlation matrices are obtained for each group.

Using network thresholding procedure described above, binary adjacency matrices for each pseudo-group over the cost range 16-25\% are 
computed (100). The C, L and BC were calculated at each cost density. The differences in network measures at each density between randomized groups are calculated. This results in a permutation distribution of differences between two randomized groups under null hypothesis. The actual difference of network measures between $\mathrm{AD}$ and $\mathrm{NL}$ groups is placed in the corresponding permutation distribution. The difference between AD and NL groups is deemed statistically significant if it lies outside $95 \%$ confidence interval of permutation distribution (two-tailed) (103).

The correlation between regional $\beta$ from FAC and their corresponding BC were evaluated by computing Pearson product-moment correlation coefficient (25). The regional BCs were averaged within 16-25\% cost range, then z-scored within each group (AD and NL), then the group difference was computed within each region. The regional $\beta$-values defined in FAC were zscored and the difference in z-scored $\beta$-values was computed between AD vs. NL groups.

\subsection{Longitudinal Changes in FDG SUR}

To examine the metabolic relevance of the regions identified as 'AD related pathological hubs' in $\mathrm{BC}$ measures, we investigated the longitudinal changes in FDG standard uptake ratios (SUR) referenced to WM mean in PAD, 
sMCI and NL subjects who were repeatedly scanned over the period of 2 years. To examine the comparative metabolic changes in regions according to their 'hubness' as observed in AD network, we divided regions into three groups based on their BC: regions with significantly high hubness (normalized difference in $\mathrm{BC}$ of $\mathrm{ROI}>2$ ), regions with significantly low hubness (normalized difference in $\mathrm{BC}$ of $\mathrm{ROI}<-2$ ) and regions with non-significant change in hubness $(\mid$ normalized difference in BC of ROI $\mid<2)$. Similarly based on their $\beta$ values and Bonferroni corrected $p$-values for the analyses, regions were classified as hypermetabolic, hypometabolic and metabolically non significant regions.

To examine the differences in FDG-SUR between regions identified as 'hubs' in $\mathrm{AD}$ progression as compared to 'non-hub' regions, we evaluated interaction effect of ROI group and time using repeated measures analysis of variance (RM-ANOVA)_in PAD, sMCI and NL groups separately. To examine the effects of using different brain parcellation scheme on FAC and graph theory network measures, we repeated all analyses with a 268-node functional atlas (27). The parcellation image is publicly available on the BioImage Suite NITRC page (https://www.nitrc.org/frs/?group_id=51). 


\section{Chapter 4: Results}

In this chapter, results of various analyses conducted in the study are provided.

\subsection{FDG PET-based AD Classifier (FAC)}

Using a GLM linear regression analysis on $88 \mathrm{AD}$ patients and 88 agesex matched healthy controls, we derived voxel-based and ROI-based FAC. The $\beta$ map is characterized by decline in FDG uptake (or hypometabolism) in medial-frontal lobes, posterior cingulum, precuneus, inferior-parietal and temporal lobes (Fig. 3). It also shows increased FDG uptake (hypermetabolism) in cerebellum, paracentral lobule and pons (Fig. 3).

The voxel-based FAC demonstrated excellent sensitivity (87.50\%) and specificity (82.95\%) in classification of AD vs NL subjects. This was preserved in 10-fold cross-validation with high sensitivity (84.09\%) and specificity (80.95\%). ROI-based FAC had almost identical metabolic topology with high topographical similarity between two classifiers ( $r=0.9720, p=8.92 \mathrm{E}-75)$. Slightly less performance was achieved with ROI-based FAC (sensitivity of $71.59 \%$ and specificity of $86.39 \%$; 10 -fold cross-validation sensitivity of $79.55 \%$ and specificity of $77.27 \%$ ). 

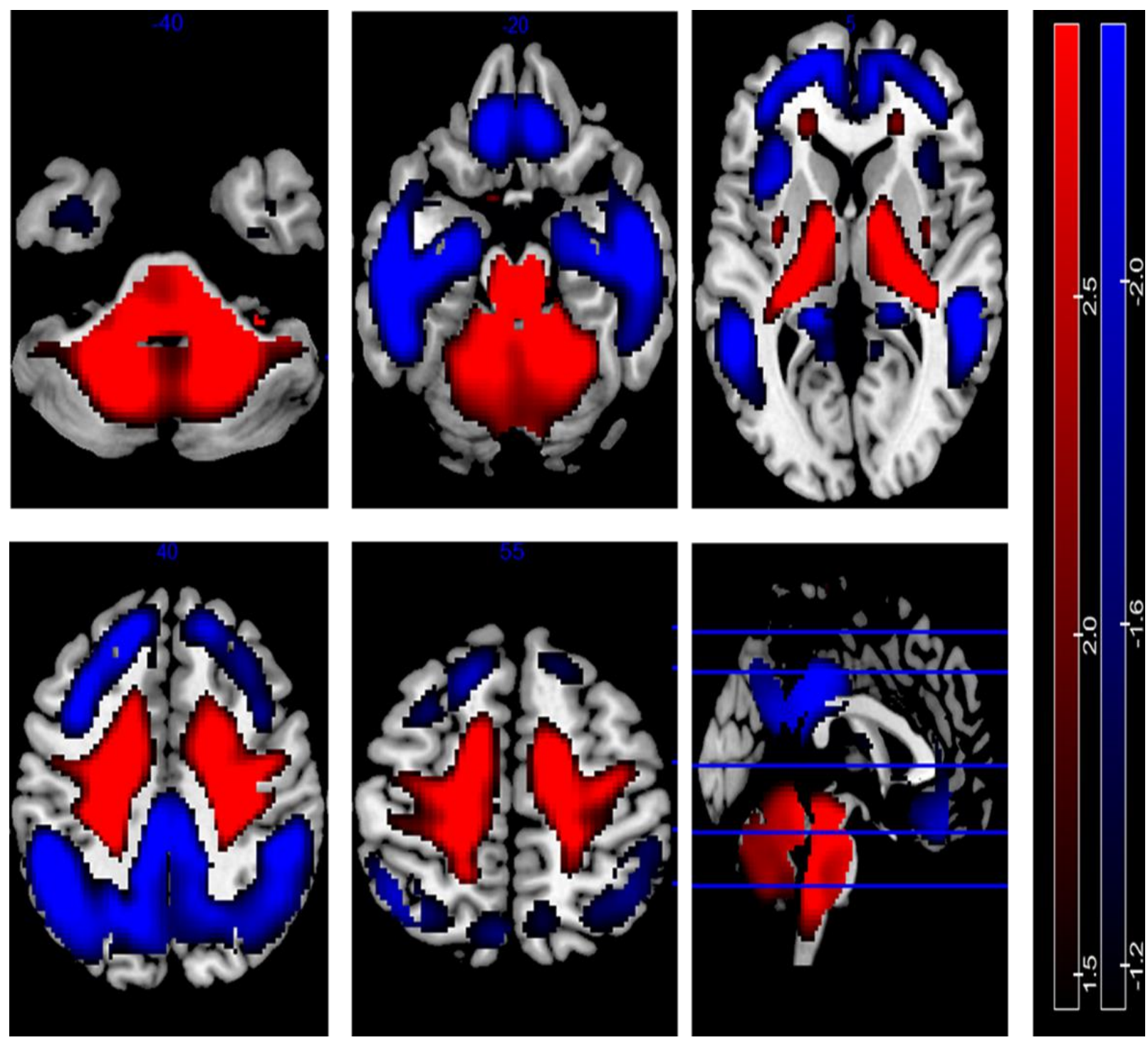

Figure3: FDG PET-based AD Classifier (FAC). AD classifier FAC is characterized by AD-like hypometabolism (shown in blue) in medial-frontal lobes, posterior cingulum, precuneus, inferior-parietal and temporal lobes. Hypermetabolism (shown in red) is observed in cerebellum, paracentral lobule and pons in $\mathrm{AD}$. The voxel-wise coefficient (voxel-weights of FAC) was zscored to the mean and standard deviation of the whole-brain. 


\subsection{AD Metabolic Network Profile}

Overall, the L in AD metabolic network was lower than the L in NL1 metabolic network over the network density range of 16\%-25\% (Fig. 4A left). The decrease in $\mathrm{L}$ was statistically significant over the examined network density range by 1000 permutations (Fig. 4A right). This is indicative of increased global efficiency in AD network for information transfer. On the contrary, $\mathrm{C}$ in $\mathrm{AD}$ metabolic network was not statistically different from the $\mathrm{C}$ in NL1 metabolic network over the network density range of 16\%-25\% (Fig. 4B). 

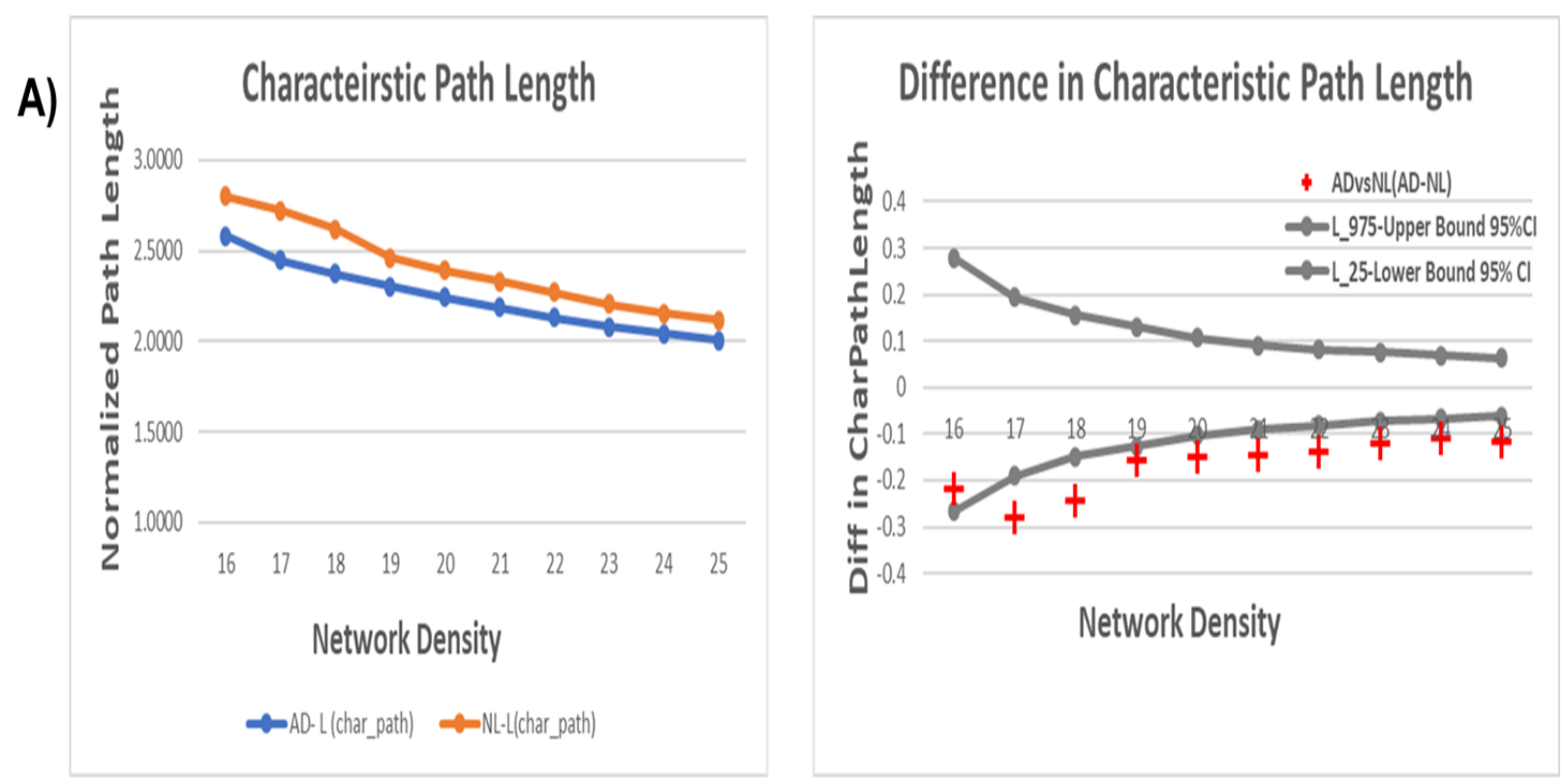

B)
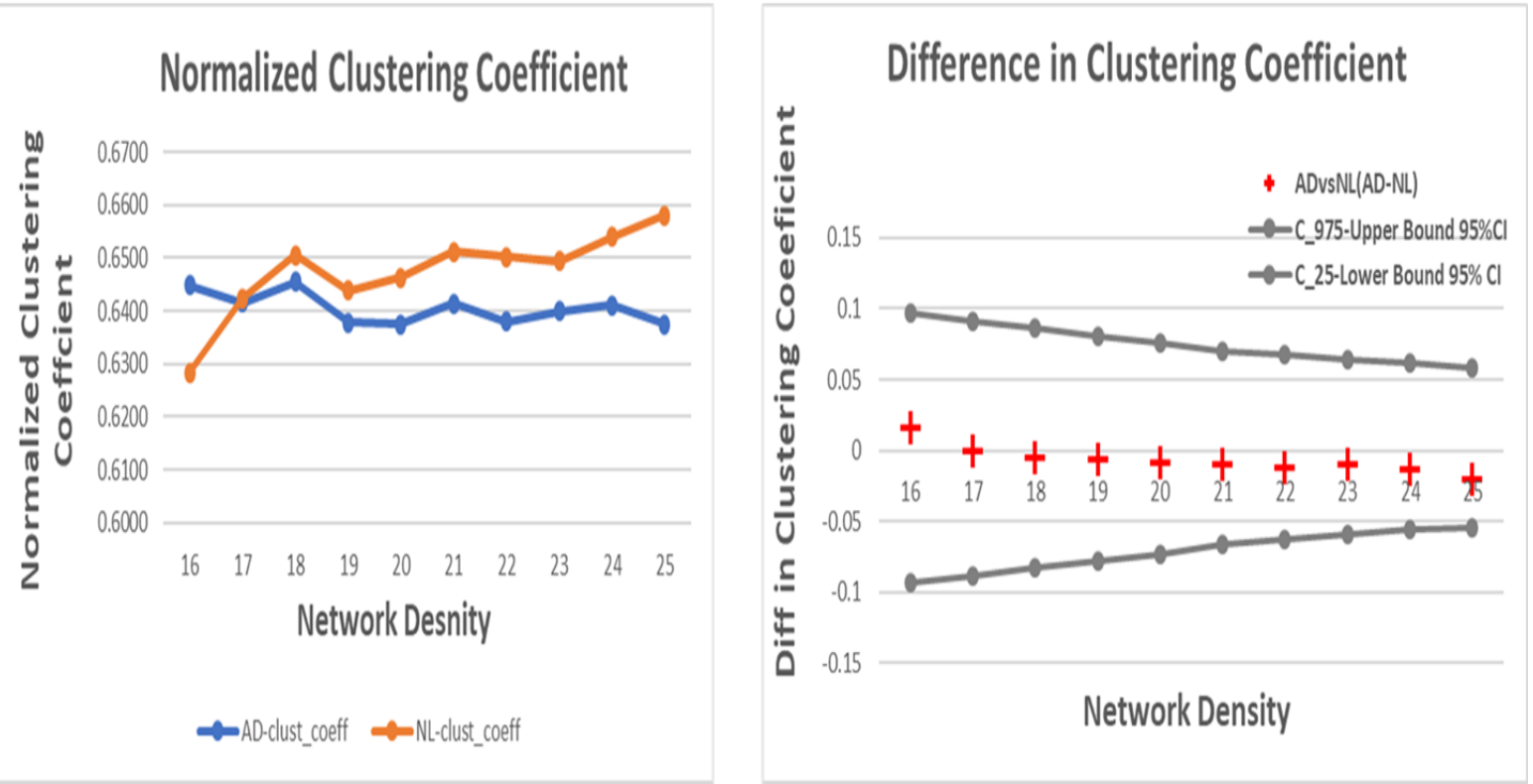

Figure 4: Changes in Global network metrics in AD network and NL1 network as a function of network cost (left). A) The changes in Characteristic Path Length (L) of AD and NL1 network over the network density range of 1625\%. B) The changes in Normalized Clustering Coefficient (C) of AD and NL1 
network over the cost range of 16-25\%. Between Group Differences in AD and NL1 network global metrics as a function of network density (right). The $95 \%$ confidence interval determined by 1000 permutation test and betweengroup differences in Characteristic Path Length (top) and Clustering Coefficient (bottom). The + marker shows the difference between NL1 and AD network (i.e $\mathrm{NL1} 1_{\text {metric }}-\mathrm{AD}_{\text {metric }}$ ), the + marker falling outside the confidence interval shows network densities at which between-group differences are significant. The positive values of + indicates $N L 1_{\text {metric }}>\mathrm{AD}_{\text {metric }}$, while the negative values of + indicates $\mathrm{NL} 1_{\text {metric }}<\mathrm{AD}_{\text {metric }}$. 


\subsection{Hubness correlate with abnormal AD metabolic topography}

Regional analysis revealed that the cerebellum, paracentral lobule, mid temporal pole and lingual gyrus showed significantly higher $\mathrm{BC}$ in $\mathrm{AD}$ network than NL network. Conversely, caudate, inferior occipital, mid-occipital and mid-temporal had significantly lower BC in AD network as compared to $\mathrm{NL}$ network. These group differences in $\mathrm{BC}$ were significantly correlated with $\beta$ in FAC ( $r=0.254, p<0.005)$ (Fig. 5). This suggests that the hub regions (high BC) of $\mathrm{AD}$ brain network generally demanded more energy consumption while the peripheral brain regions (low $\mathrm{BC}$ ) in the $\mathrm{AD}$ brain network was associated with decreased glucose metabolism.

The relationship between hubness and abnormal $\mathrm{AD}$ metabolic topography was replicated when a 268-node functional atlas was used as an alternate parcellation scheme $(\mathrm{r}=0.125, \mathrm{p}=0.04)$ (Fig. 6). The functional atlas used here is produced using a groupwise graph theory based parcellation scheme for node definition in network analysis. This suggests that this relationship is independent of how brain regions are defined in a brain parcellation atlas. 


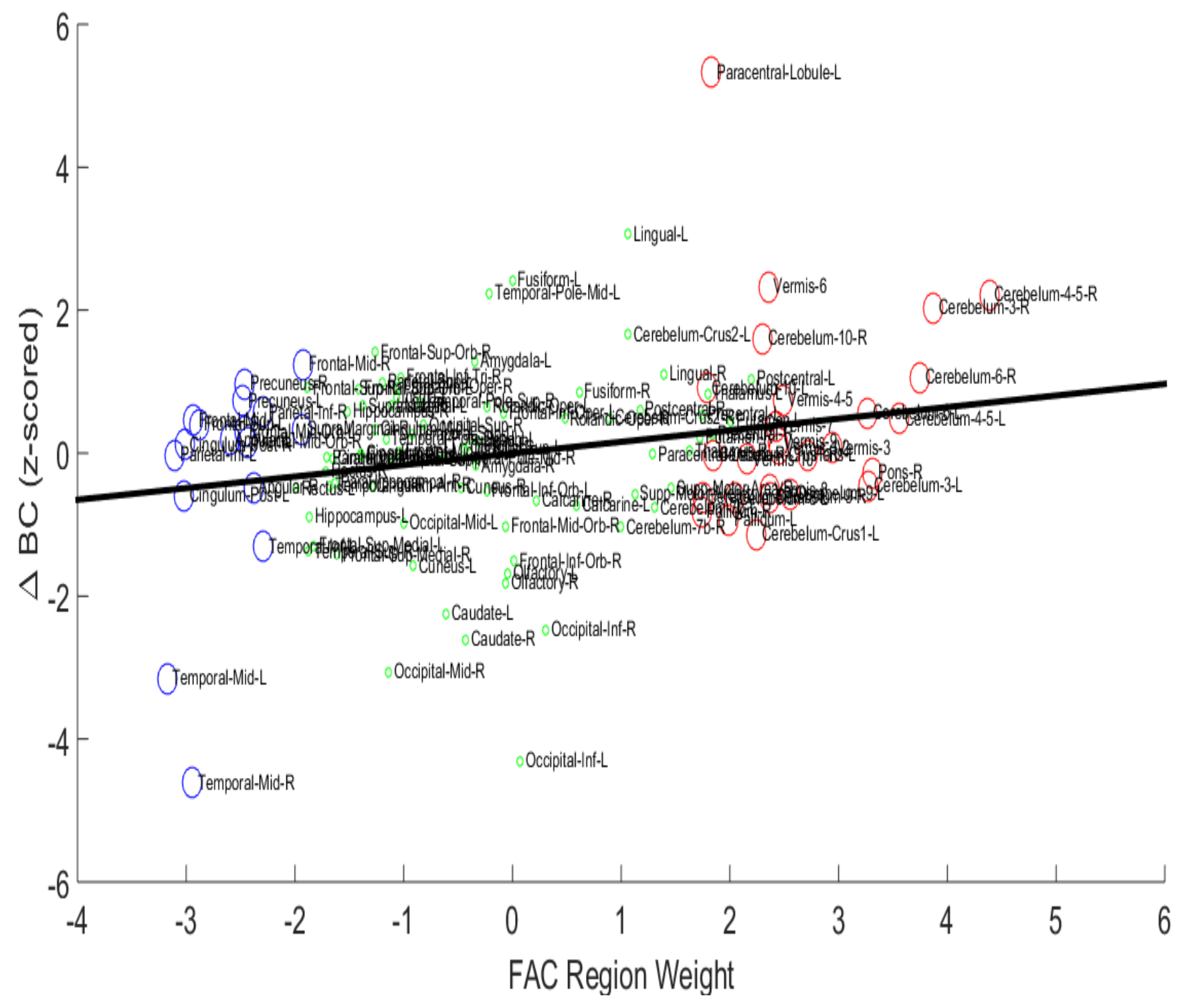

Figure 5: Correlation between FAC Region Weights and Betweenness

Centrality of regions. The region weights of regions on FAC are plotted on $\mathrm{x}-$ axis, while the normalized difference in $\mathrm{BC}$ between $\mathrm{AD}$ and NL network is plotted on y-axis. The regions are delineated using AAL (26). The regions shown by red circles are identified as hypermetabolic on AD metabolic pattern, 
while the ones shown in blue are identified as hypometabolic on FAC. The regions denoted by green circles are the ones which do not undergo significant metabolic change in AD. Significant correlation $(r=0.254, \mathrm{p}<0.005)$, shown by solid black line, exists between region weights of regions on FAC and their corresponding $\mathrm{BC}$ values. 


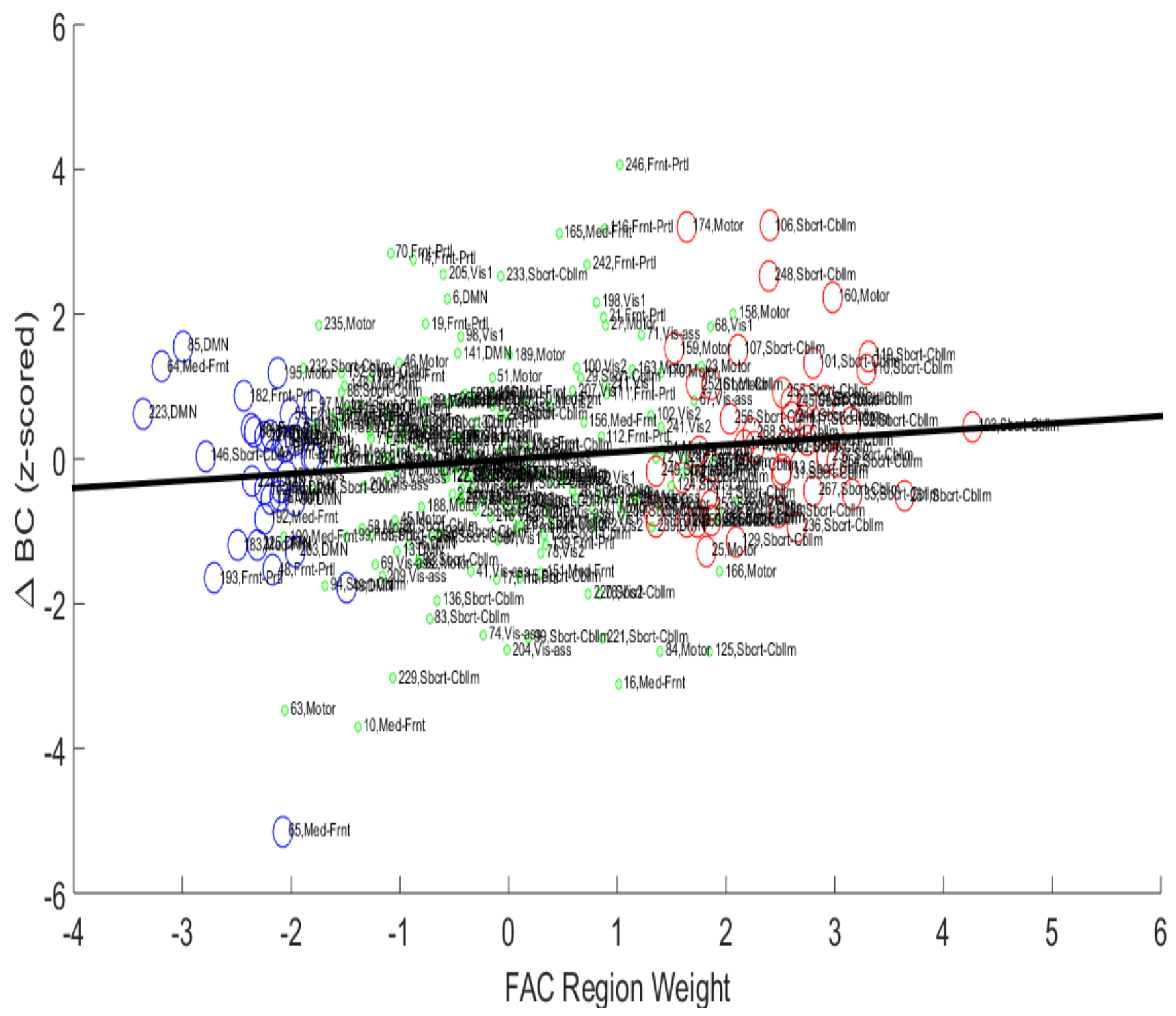

Figure 6: Effect of Brain Parcellation Scheme on Relationship between FAC

Region Weights and Betweenness Centrality. The region weights of regions identified from 268 region Atlas (27) on FAC are plotted on x-axis, while the normalized difference in $\mathrm{BC}$ for these regions between $\mathrm{AD}$ and NL network is 
plotted on y-axis. The regions shown by red circles are identified as hypermetabolic on $\mathrm{AD}$ metabolic pattern, while the ones shown in blue are identified as hypometabolic on FAC. The regions denoted by green circles are the ones which do not undergo significant metabolic change in AD. Correlation $(\mathrm{r}=0.125, \mathrm{p}=0.04)$, shown by solid black line, exists between region weights of regions on FAC and their corresponding $\mathrm{BC}$ values. This suggests that the relationship between $\mathrm{FAC}$ region weights and their corresponding $\mathrm{BC}$ values is independent of brain parcellation scheme. 


\subsection{Longitudinal Changes of FDG-SUR in Hub vs Non-Hub Regions}

We investigated the longitudinally measured changes in FDG-SUR to compare metabolism in the regions with high $\triangle \mathrm{BC}$, regions with non-significant $\triangle \mathrm{BC}$ and regions with low $\triangle \mathrm{BC}$ in NL, sMCI and PAD. Significant main effects of time were observed in all three groups (NL, sMCI, and PAD; $\mathrm{p}<0.001)$. Significant interaction effect between time and brain regions (divided by BC) were observed only in PAD $(\mathrm{F}(4,152)=10.962, \mathrm{p}=7.764 \mathrm{E}-08$, Fig. 7). In particular, the hub regions of $\mathrm{AD}$ network with high $\mathrm{BC}$ showed increased metabolism over time ( $\mathrm{p}=0.001$, post-hoc Bonferroni). The metabolic changes in regions with low BC and non significant BC were not significant $(p>0.147$, post-hoc Bonferroni). No significant interaction effect was observed between time and brain regions in $\mathrm{NL}(\mathrm{F}(2,73)=1.144, \mathrm{p}=0.324)$ or $\mathrm{sMCI}$ $(\mathrm{F}(4,152)=0.706, \mathrm{p}=0.589)$. 
A)

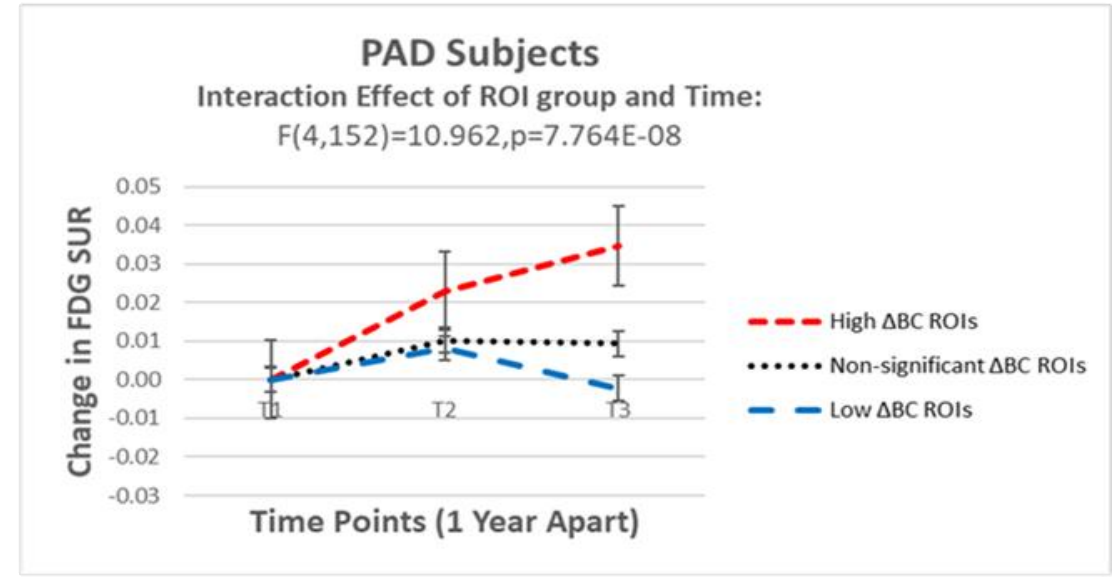

B)

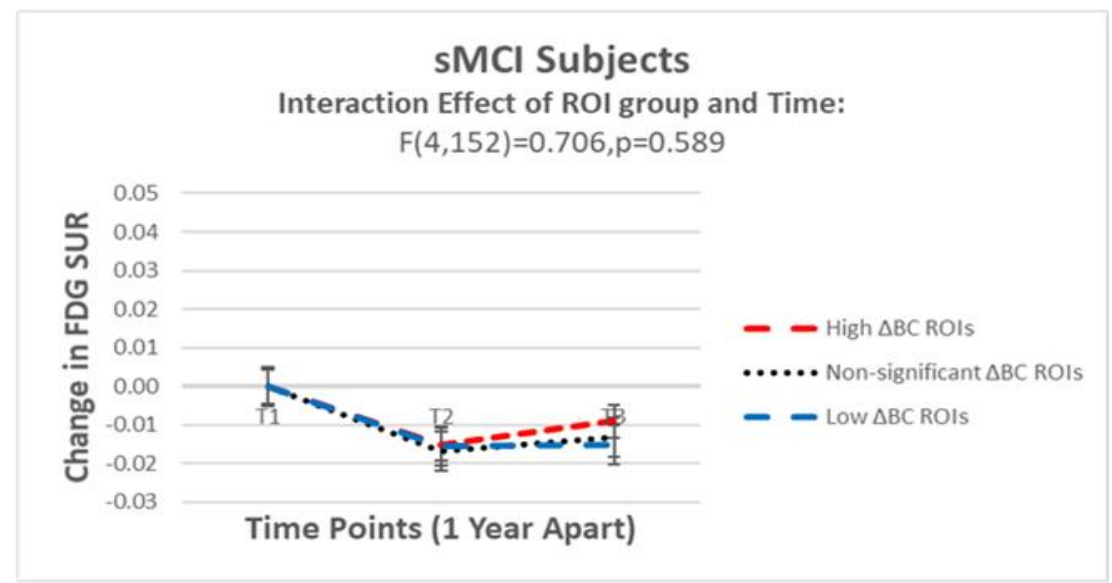

C)

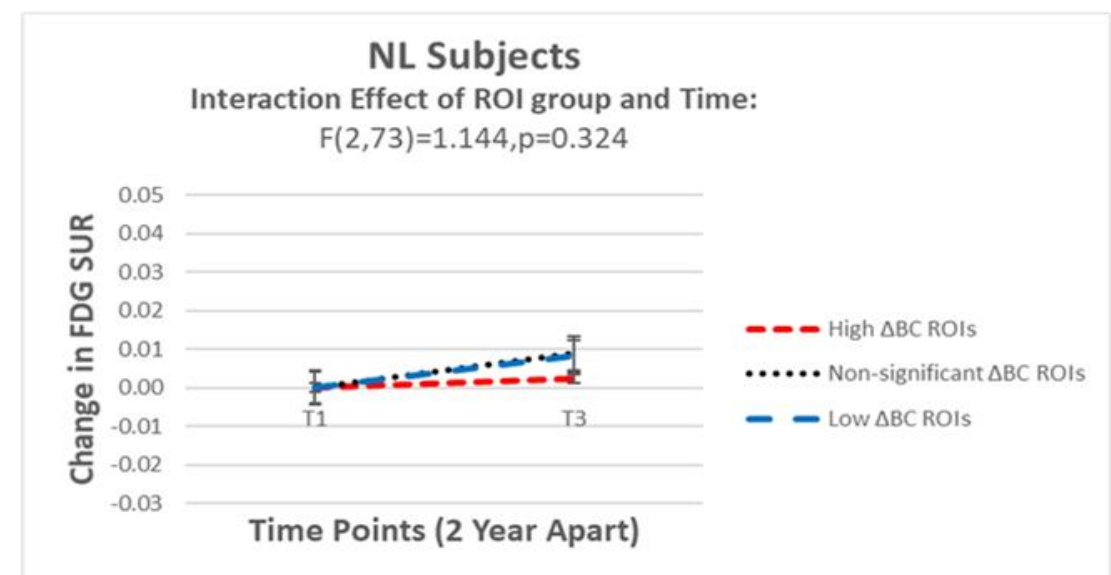

Figure 7: Longitudinal Changes in FDG-SUR. The overall averaged changes in FDG-SUR in the high $\triangle \mathrm{BC}$ regions (shown by dotted red line), nonsignificant $\triangle \mathrm{BC}$ regions (shown by dotted black line) and low $\triangle \mathrm{BC}$ regions 
(shown by dotted blue line). The high, non-significant, and low $\triangle \mathrm{BC}$ regions were determined based on $\mathrm{BC}$ differences between $\mathrm{AD}$ vs. NL1 shown in Figure $5(\Delta \mathrm{BC}>2,-2<\Delta \mathrm{BC}<2, \Delta \mathrm{BC}<-2$, respectively). $\mathrm{A})$ In the PAD subjects, there is significant difference in averaged FDG-SUR between three groups with a significant interaction effect of ROI group and time $(F(4,152)=10.962$, $\mathrm{p}=7.764 \mathrm{E}-08$ ). The metabolism increases significantly in 'hub' regions in PAD subjects near conversion to $\mathrm{AD}$. B) In the $\mathrm{sMCI}$ subjects, there is no significant difference in averaged FDG-SUR between three ROI groups $(F(4,152)=0.706$, $\mathrm{p}=0.589$ ). C) In the NL2 subjects, there is no significant interaction effect of ROI group and time $(\mathrm{F}(2,73)=1.144, \mathrm{p}=0.324)$. 


\section{Chapter 5: Discussion}

Conventional practices of FDG PET reading for $\mathrm{AD}$ diagnosis have focused on hypometabolism in key anatomical regions. Hypermetabolism observed in $\mathrm{AD}$ is often dismissed as an artefact arising due to use of global mean for normalization in traditional studies (17). There is increasing evidence from anatomical and neuroimaging studies which is leading to a paradigm shift in the role of hypermetabolic regions in neurodegenerative diseases (19). Using whole brain WM mean for signal normalization, we confirmed that hypermetabolism observed in AD is not an artefact merely introduced by the global signal normalization.

Emerging evidence suggests that the hypermetabolism observed in $\mathrm{AD}$ patients is an integral part of AD pathophysiology (18). This calls for inclusion of cerebellar hypermetabolism while deriving biomarkers for $\mathrm{AD}$ diagnosis. In our study, we derived FAC as AD classifier using whole brain WM mean. FAC has high sensitivity of $87.50 \%$ and specificity of $82.95 \%$, and it has been demonstrated to be a robust classifier with high accuracy after 10-fold cross validation. We suspect that the performance of FAC could be improved if a final diagnosis was available so that clinically misdiagnosed patients were excluded from the analysis. 
In further graph theory analysis on the metabolic network, we observed a statistically significant decrease in $\mathrm{L}$ in $\mathrm{AD}$ metabolic network when compared to NL1, whereas the changes in $\mathrm{C}$ were not statistically significant between the two groups. L of a network is the measure of the average shortest path length between all pairs of nodes in the network (74), whereas $\mathrm{C}$ of a graph measures the cliquishness of a typical graph neighborhood and is a measure of the degree to which nodes in a graph tend to cluster together (75). The phenomena of decreasing $\mathrm{L}$ and unchanging $\mathrm{C}$ is a well described characteristic in Watts-Strogatz 'smallworld' model (75), where it was explained as such that a network becomes a small world (and more cost-efficient) when there is more "randomness" in the re-wiring process. The random rewiring of the connections in a network introduces shortcuts among nodes resulting in decreased L while maintaining $\mathrm{C}$.

An RS-fMRI study of AD subjects found that decreased normalized path length is directly associated with lower MMSE scores of patients suggesting that increase in random topology of graph is associated with worse cognitive functioning (85). This balance of short path length and high clustering in AD network suggests an evolutionary optimization of the equilibrium between information segregation (represented by nodes forming particular clusters) and 
information integration (represented by interactions among clusters) (94). The regional changes associated with this increased "randomness" (N.B. it should be noted that we are not implying that the brain network rewiring is happening in a random fashion, but it refers to the opposite of regular network in WattsStrogatz model) can be investigated by understanding which regions gained "hubness" (the new important functional node).

BC for each node quantifies hubness of that node by incorporating connectivity information from the whole network (80). Brain regions acting as hubs make information processing in the network more efficient, thereby increasing the robustness of the network towards random failure (104). Cerebellar lobules, paracentral lobule and lingual gyrus (regions defined according to AAL region definition) were the key regions associated with increased hubness in $\mathrm{AD}$ network as compared to controls. The increased hubness in these regions may reflect the adaptive role of these regions (via increased recruitment) in $\mathrm{AD}$ patients to limit the clinical consequences of loss of functionality in large scale networks associated with high level cognition in AD patients.

Recent neuroimaging and neurophysiological studies have suggested disruption in neuronal integrity of large scale functional networks associated 
with high level cognition in AD patients (105). Emerging evidence from brain imaging studies have established the integral role of the cerebellum in modulation of cognition and emotion, establishing its contribution to cognitive and neuropsychiatric deficits in $\mathrm{AD}$ (18). The cerebellum is reported to be associated with cerebral sensorimotor regions and higher-order cognitive regions via feedback projections through thalamus (106). These connections suggest cerebellar involvement in cognitive processes, and the fact that these connections retain their functional capacity in early stages of $\mathrm{AD}$ may explain the increased hubness in cerebellar regions in $\mathrm{AD}$ patients.

The paracentral lobule is associated with the control of motor and sensory innervations of the contralateral lower extremity. In light of AD-related loss in function of the primary sensorimotor regions, the increased hubness in paracentral lobule could be attributed to the increased recruitment of paracentral lobule to maintain task performance. Previous study has also reported increase in hubness of lingual gyrus (91). It is suggested that lingual gyrus retain its functional capacity in early stages of $\operatorname{AD}(107,108)$, which might explain the observed increase in hubness of lingual gyrus in $\mathrm{AD}$ network when compared to normal controls. 
We observed significant decrease in hubness of a few hypometabolic regions in $\mathrm{AD}$ network which may be a direct consequence of corticocortical dysconnectivity. These regions (middle temporal, occipitotemporal, and caudate) have been well documented to be affected in $\operatorname{AD}(109,110)$. Interestingly, the above mentioned cortical areas that lose hubness have been also related to cortical atrophy (111). Moreover, the loss of local centrality in these cortical regions has been found to be strongly related with the cognitive decline in AD patients (112). And thus, decreased BC paired with decreased metabolism in the mid-temporal region, inferior occipital region and caudate may be explained by the loss of neuronal function and potentially accompanied by atrophy.

It must be noted that the level of correlation between changes in $\mathrm{BC}$ and glucose metabolism was very weak $(\mathrm{r}=0.25)$ although it was statistically significant. The significance was primarily driven by a few hypermetabolic and hypometabolic nodes paired with increased and decreased BC, respectively, as described above. Yet, the majority of the brain regions showed preserved BC level in AD compared to NL1; while apparent metabolic changes has been consistently observed. This may be caused by relative insensitivity of $\mathrm{BC}$ measurement, the statistical significance of which was conservatively tested 
with 1,000 permutations. And, it should be also noted that the AD individuals that were included in ADNI project had very early stage AD (MMSE score ranges from 20 to 26), and thus it may be possible that these regions retained some level of functional connectivity despite decreasing neuronal glucose metabolism. For example, it has been suggested that neurons in early AD become more reliant on ketone (primary alternative fuel to glucose in the brain) compared to glucose, which is relatively preserved in early $\mathrm{AD}$.

In order to understand the functional relevance of the regions with high or low $\triangle \mathrm{BC}$ in $\mathrm{AD}$ development, the longitudinal metabolic changes were compared in PAD, sMCI, and NL2. When divided by $\triangle \mathrm{BC}$ (AD vs. NL1), the regions with high $\triangle \mathrm{BC}$ (which included cerebellar lobules, left lingual gyri, paracentral lobule) are the only regions that showed significant metabolic changes (increased) over the two years of follow-up period in PAD which was not observed in sMCI and NL2. This suggests that the progressive regional metabolic increases associated with increased hubness precedes AD diagnosis at least by one year and that it may be involved with rapid symptom worsening that can be observed one year prior to the clinical diagnosis. 


\section{Limitations}

Graph theory is being rigorously applied to derive and investigate brain networks obtained with anatomical and functional neuroimaging modalities. It is reasonable to assume that if brain networks are stable across neuroimaging techniques, then they should be consistent in their network topologies (87). Reviews on graph theoretic studies in AD patients have reported discrepancies among studies (85), which suggests the need to investigate that whether connectivity interpreted across various neuroimaging techniques refer to the same underlying construct.

Although models based on graph theory explain important properties of brain networks in neurodegenerative diseases, they don't predict what happens when neuronal connections are lost, which is characteristic of a neurodegenerative disease like AD (94). The interpretation of increased characteristic path length in $\mathrm{AD}$ network remains ambiguous as considerable variability exists across studies regarding characteristic path length $(85,92)$. These diverging results across imaging modalities might imply that brain graphs are not isomorphic across various modalities and different modalities measure different aspects of brain graphs. 
A possible confounder in graph theory-based models of brain graphs is the lack of a standardized method to construct graphs. The differences in sizes and connectivity densities of nodes used to define a graph have been shown to influence graph metrics, so node definitions should therefore be kept constant across studies to enable comparing results (101). Moreover, the interpretation of graph theory based metrics is further complicated by node definitions, as nodes are comprised of different amount of cortical tissues, resulting in differences in signal to noise ratios (113).

\section{Future Perspectives}

The use of graph theory to model disease network in AD and understand disease progression is still in early phase. Studies have established that AD patients have disruptive neuronal integrity in large scale brain networks associated with cognition, motor control and memory formation. The next step in modelling $\mathrm{AD}$ networks and understanding disease progression is to formulate a hypothesis generating framework to understand the role of altered topologies in cognitive dysfunctioning.

The models developed in graph theory for analysis of brain networks in neurodegenerative diseases like $\mathrm{AD}$ are developmental models; they are aimed at predicting what happens when connections are added or rewired; they do not 
necessarily predict the effect of removing connections which is characteristic of a neurodegenerative disease. Future work is needed towards defining more elegant models to generate hypotheses which allow us to investigate and predict the effect of rewiring connections, as well as loss of connections, in a network.

There is significant potential for application of graph theory in early diagnosis of $\mathrm{AD}$, especially in MCI patients who have increased risk to develop AD. Studies have suggested that topology of MCI graphs is intermediate between healthy subjects and AD patients. However, significant, but subtle, differences have been observed when comparing MCI subjects with normal controls suggesting that larger samples are required when comparing these three groups. 


\section{Chapter 6: Conclusion}

Our study established that hypermetabolism observed in AD is not an artefact of using global mean in signal normalization; instead it suggests that hypermetabolism might be integral component of $\mathrm{AD}$ pathology. There is rich, but under-appreciated literature, documenting cerebellar hypermetabolism in $\mathrm{AD}$ and role of cerebellum in AD pathology. This study suggests that more research is needed to understand cause of hypermetabolism in $\mathrm{AD}$ and potentially investigate the role of cerebellum in neuropathology and disease progression in $\mathrm{AD}$. The classifier FAC established using whole brain WM mean had high sensitivity and specificity in separating AD patients from normal controls.

The disruption in large scale structural and functional neuronal networks in $\mathrm{AD}$ causes the network to become more random, resulting in an evolutionary optimization of the balance of information segregation and information integration. Within this framework, we propose that cerebellum is more than a silent bystander in the pathophysiology of $\mathrm{AD}$. We suggest a robust model where the loss of hubness in cortical regions observed in $\mathrm{AD}$ patients is paralleled by increase in hubness of cerebellar regions, with the possible 
adaptive role to limit the clinical consequences of tissue damage associated with AD. 


\section{References:}

1. Gaugler J, James B, Johnson T, Marin A, Weuve J. 2019 Alzheimer's disease facts and figures. Alzheimer's \& Dementia. 2019;15(3):321-87. doi: 10.1016/j.jalz.2019.01.010.

2. 2018 Alzheimer's disease facts and figures. Alzheimer's \& Dementia. 2018;14(3):367-429. doi: 10.1016/j.jalz.2018.02.001.

3. Sperling RA, Aisen PS, Beckett LA, Bennett DA, Craft S, Fagan AM, et al. Toward defining the preclinical stages of Alzheimer's disease: Recommendations from the National Institute on Aging-Alzheimer's Association workgroups on diagnostic guidelines for Alzheimer's disease. Alzheimer's \& Dementia: The Journal of the Alzheimer's Association. 2011;7(3):280-92. doi: 10.1016/j.jalz.2011.03.003.

4. Dá Mesquita S, Ferreira AC, Sousa JC, Correia-Neves M, Sousa N, Marques F. Insights on the pathophysiology of Alzheimer's disease: The crosstalk between amyloid pathology, neuroinflammation and the peripheral immune system. Neuroscience and Biobehavioral Reviews. 2016;68:547-62. doi: 10.1016/j.neubiorev.2016.06.014. 
5. Imbimbo BP, Lombard J, Pomara N. Pathophysiology of Alzheimer's disease. Neuroimaging Clinics of North America. 2005;15(4):727-53. doi: 10.1016/j.nic.2005.09.009.

6. Lin TP, Carbon M, Tang C, Mogilner AY, Sterio D, Beric A, et al. Metabolic correlates of subthalamic nucleus activity in Parkinson's disease. Brain. 2008;131(5):1373-80. doi: 10.1093/brain/awn031.

7. McConathy J, Sheline YI. Imaging Biomarkers Associated With Cognitive Decline: A Review. Biological Psychiatry. 2015;77(8):685-92. doi: 10.1016/j.biopsych.2014.08.024.

8. Audrey K, Paul S, Andrew LG, Daniel L, Bohdan B, Maram A, et al. Machine learning identified an Alzheimer's disease-related FDG-PET pattern which is also expressed in Lewy body dementia and Parkinson's disease dementia. Scientific Reports. 2018;8(1):1-13. doi: 10.1038/s41598-018-31653-6.

9. Laura K. Teune FS, Remco J. Renken, Gerbrand J. Izaks, Jeroen J. de Vries, Marcel Segbers, Jos B.T.M. Roerdink, Rudi A.J.O. Dierckx and Klaus L. Leenders. The Alzheimer's Disease-Related Glucose Metabolic Brain Pattern. Current Alzheimer Research. 2014;11:725. doi: 10.2174/156720501108140910114230. 
10. Yamane T, Ikari Y, Nishio T, Ishii K, Kato T, Ito K, et al. VisualStatistical Interpretation of F-18-FDG-PET Images for Characteristic Alzheimer Patterns in a Multicenter Study: Inter-Rater Concordance and Relationship to Automated Quantitative Evaluation. American Journal Of Neuroradiology. 2014;35(2):244-9. doi: 10.3174/ajnr.A3665.

11. Toga AW, Crawford KL. The Alzheimer's Disease Neuroimaging Initiative informatics core: A decade in review. Alzheimer's \& Dementia: The Journal of the Alzheimer's Association. 2015;11(7):832-9. doi: 10.1016/j.jalz.2015.04.004.

12. Partovi S, Yuh R, Pirozzi S, Lu Z, Couturier S, Grosse U, et al. Diagnostic performance of an automated analysis software for the diagnosis of Alzheimer's dementia with F FDG PET. American journal of nuclear medicine and molecular imaging. 2017;7(1):12.

13. Shaffer JL, Petrella JR, Sheldon FC, Choudhury KR, Calhoun VD, Coleman RE, et al. Predicting cognitive decline in subjects at risk for Alzheimer disease by using combined cerebrospinal fluid, MR imaging, and PET biomarkers. Radiology. 2013;266(2):583-91. doi: 10.1148/radiol.12120010. 
14. Chen X, Zhou Y, Wang R, Cao H, Reid S, Gao R, et al. Potential Clinical Value of Multiparametric PET in the Prediction of Alzheimer's Disease $\begin{array}{llll}\text { Progression. } & \text { PLoS } & \text { ONE. }\end{array}$ 10.1371/journal.pone.0154406.

15. Zhang D, Shen D. Predicting Future Clinical Changes of MCI Patients Using Longitudinal and Multimodal Biomarkers (Predicting Future Clinical Changes of MCI Patients). PLoS ONE. 2012;7(3):e33182. doi: 10.1371/journal.pone.0033182.

16. Buchert R, Wilke F, Chakrabarti B, Martin B, Brenner W, Mester J, et al. Adjusted scaling of FDG positron emission tomography images for statistical evaluation in patients with suspected Alzheimer's disease. Journal Of Neuroimaging. 2005;15(4):348-55. doi: $10.1177 / 1051228405280169$.

17. Borghammer P, Cumming P, Aanerud J, Gjedde A. Artefactual subcortical hyperperfusion in PET studies normalized to global mean: Lessons from Parkinson's disease. NeuroImage. 2009;45(2):249-57. doi: 10.1016/j.neuroimage.2008.07.042.

18. Jacobs HIL, Hopkins DA, Mayrhofer HC, Bruner E, van Leeuwen FW, Raaijmakers W, et al. The cerebellum in Alzheimer's disease: evaluating 
its role in cognitive decline. Brain : a journal of neurology. 2018;141(1):37-47. doi: 10.1093/brain/awx194.

19. Miyazawa N, Shinohara T, Nagasaka T, Hayashi M. Hypermetabolism in Patients With Dementia With Lewy Bodies. Clinical Nuclear Medicine. 2010;35(7):490-3. doi: 10.1097/RLU.0b013e3181e05dbc.

20. Chilumuri A, Ashioti M, Nercessian AN, Milton NGN. Immunolocalization of Kisspeptin Associated with Amyloid- Deposits in the Pons of an Alzheimer's Disease Patient. Journal of Neurodegenerative Diseases. 2013;2013. doi: 10.1155/2013/879710.

21. Jansen F, Stam C, Braun K, Otte W. Brain Network Organization in Focal Epilepsy: A Systematic Review and Meta-Analysis. PLoS One. 2014;9(12):e114606. doi: 10.1371/journal.pone.0114606.

22. Khazaee A, Ebrahimzadeh A, Babajani-Feremi A. Identifying patients with Alzheimer's disease using resting-state fMRI and graph theory. Clinical Neurophysiology. 2015;126(11):2132-41. doi: 10.1016/j.clinph.2015.02.060.

23. Spetsieris P, Ma Y, Peng S, Ko JH, Dhawan V, Tang CC, et al. Identification of disease-related spatial covariance patterns using neuroimaging data. Journal of visualized experiments : JoVE. 2013(76). 
24. Selkoe DJ. Early network dysfunction in Alzheimer's disease. Science (New York, NY). 2019;365(6453):540. doi: 10.1126/science.aay5188.

25. Ko JH, Spetsieris PG, Eidelberg D. Network Structure and Function in Parkinson's Disease. Cerebral Cortex. 2018;28(12):4121-35. doi: 10.1093/cercor/bhx267.

26. Lancaster J, Woldorff M, Parsons L, Liotti M, Freitas C, Rainey L, et al. Automated Talairach Atlas Labels For Functional Brain Mapping. Human Brain Mapping. 2000;10(3):120-31. doi: 10.1002/10970193(200007)10:3<120::AID-HBM30>3.3.CO;2-\#.

27. Emily SF, Xilin S, Dustin S, Monica DR, Jessica H, Marvin MC, et al. Functional connectome fingerprinting: identifying individuals using patterns of brain connectivity. Nature Neuroscience. 2015;18(11). doi: 10.1038/nn.4135.

28. Shen X, Tokoglu F, Papademetris X, Constable RT. Groupwise wholebrain parcellation from resting-state fMRI data for network node identification. NeuroImage. 2013;82:403-15. doi: 10.1016/j.neuroimage.2013.05.081. 
29. Qiu C, De Ronchi D, Fratiglioni L. The epidemiology of the dementias: an update. Current Opinion in Psychiatry. 2007;20(4):380-5. doi: 10.1097/YCO.0b013e32816ebc7b.

30. Katzman R. The Prevalence and Malignancy of Alzheimer Disease: A Major Killer. Archives of Neurology. 1976;33(4):217-8. doi: 10.1001/archneur.1976.00500040001001.

31. Lancet. A global assessment of dementia, now and in the future. 2015;386(9997):931-. doi: 10.1016/S0140-6736(15)00117-8.

32. Braak RH, Thal RD, Ghebremedhin RE, Del Tredici RK. Stages of the Pathologic Process in Alzheimer Disease: Age Categories From 1 to 100 Years. Journal of Neuropathology and Experimental Neurology. 2011;70(11):960-9. doi: 10.1097/NEN.0b013e318232a379.

33. Christiane R, Carol B, Richard M. Epidemiology of Alzheimer disease. $\begin{array}{llll}\text { Nature } & \text { Reviews } & \text { Neurology. 2011;7(3):137. doi: }\end{array}$ 10.1038/nrneurol.2011.2.

34. Wang H-X, Xu W, Pei J-J. Leisure activities, cognition and dementia. BBA - Molecular Basis of Disease. 2012;1822(3):482-91. doi: 10.1016/j.bbadis.2011.09.002. 
35. Saczynski JS, Pfeifer LA, Masaki K, Korf ESC, Laurin D, White L, et al. The Effect of Social Engagement on Incident Dementia. American Journal of Epidemiology. 2006;163(5):433-40. doi: 10.1093/aje/kwj061.

36. Christian H, Dennis JS. Soluble protein oligomers in neurodegeneration: lessons from the Alzheimer's amyloid $\beta$-peptide. Nature Reviews Molecular Cell Biology. 2007;8(2):101. doi: 10.1038/nrm2101.

37. Bischof GN, Jessen F, Fliessbach K, Dronse J, Hammes J, Neumaier B, et al. Impact of tau and amyloid burden on glucose metabolism in Alzheimer's disease. Annals of Clinical and Translational Neurology. 2016;3(12):934-9. doi: 10.1002/acn3.339.

38. Hardy J. The amyloid hypothesis for Alzheimer's disease: a critical reappraisal. Oxford, UK: Blackwell Publishing Ltd; 2009. p. 1129-34.

39. Hardy J, Selkoe D. The Amyloid Hypothesis of Alzheimer's Disease: Progress and Problems on the Road to Therapeutics. Science (Washington). 2002;297(5580):353-6.

40. Mosconi L, Berti V, Glodzik L, Pupi A, De Santi S, de Leon M. PreClinical Detection of Alzheimer's Disease Using FDG-PET, with or without Amyloid Imaging. Journal Of Alzheimers Disease. 2010;20(3):843-54. doi: 10.3233/JAD-2010-091504. 
41. Neuropathology Group. Medical Research Council Cognitive Function and Aging S. Pathological correlates of late-onset dementia in a multicentre, community-based population in England and Wales. The Lancet. 2001;357(9251):169-75. doi: 10.1016/S0140-6736(00)03589-3.

42. Gómez-Isla T, Hollister R, West H, Mui S, Growdon JH, Petersen RC, et al. Neuronal loss correlates with but exceeds neurofibrillary tangles in Alzheimer's disease. Annals of Neurology. 1997;41(1):17-24. doi: 10.1002/ana.410410106.

43. Knopman D, Parisi J, Salviati A, Floriach-Robert M. Neuropathology of cognitively normal elderly. Journal of Neuropathology and Experimental Neurology. 2003;62(11):1087-95. doi: 10.1093/jnen/62.11.1087.

44. Petersen R. Early Diagnosis of Alzheimers Disease: Is MCI Too Late? Current Alzheimer Research. 2009;6(4):324-30. doi: $10.2174 / 156720509788929237$.

45. Benson AD, Slavin MJ, Tran TT, Petrella JR, Doraiswamy PM. Screening for early Alzheimer's disease: Is there still a role for the MiniMental State Examination? Primary Care Companion to the Journal of Clinical Psychiatry. 2005;7(2):62-7. 
46. Viswanathan AA, Rocca AW, Tzourio AC. Vascular risk factors and dementia: How to move forward? Neurology. 2009;72(4):368-74. doi: 10.1212/01.wnl.0000341271.90478.8e.

47. Williams MM, Roe CM, Morris JC. Stability of the Clinical Dementia Rating, 1979-2007. Archives of Neurology. 2009;66(6):773-7. doi: 10.1001/archneurol.2009.69.

48. Zhou Y, Ortiz F, Nuñez C, Elashoff D, Woo E, Apostolova LG, et al. Use of the MoCA in Detecting Early Alzheimer's Disease in a SpanishSpeaking Population with Varied Levels of Education. Dementia and Geriatric Cognitive Disorders Extra. 2015;5(1):85-95. doi: $10.1159 / 000365506$.

49. Teng WE, Becker SB, Woo LE, Knopman HD, Cummings HJ, Lu HP. Utility of the Functional Activities Questionnaire for Distinguishing Mild Cognitive Impairment From Very Mild Alzheimer Disease. Alzheimer Disease \& Associated Disorders. 2010;24(4):348-53. doi: 10.1097/WAD.0b013e3181e2fc84.

50. Sokoloff L. Function-related Changes in Energy Metabolism in the Nervous System: Localization and Mechanisms. The Keio Journal of Medicine. 1993;42(3):95-103. doi: 10.2302/kjm.42.95. 
51. Salomone S, Caraci F, Leggio GM, Fedotova J, Drago F. New pharmacological strategies for treatment of Alzheimer's disease: Focus on disease modifying drugs. British Journal of Clinical Pharmacology. 2012;73(4):504-17. doi: 10.1111/j.1365-2125.2011.04134.x.

52. Cacabelos R. Donepezil in Alzheimeras disease: From conventional trials to pharmacogenetics. Neuropsychiatric Disease and Treatment. 2007;3(3):303-33.

53. Deardorff WJ, Grossberg GT. A fixed-dose combination of memantine extended-release and donepezil in the treatment of moderate-to-severe alzheimer's disease.(Report). Drug Design, Development and Therapy. 2016;10:3267. doi: 10.2147/DDDT.S86463.

54. Zhou X, Li Y, Shi X, Ma C. An overview on therapeutics attenuating amyloid $\beta$ level in Alzheimer's disease: targeting neurotransmission, inflammation, oxidative stress and enhanced cholesterol levels. American journal of translational research. 2016;8(2):246-69.

55. Aguirre E, Woods R, Spector A, Orrell M. Cognitive stimulation for dementia: A systematic review of the evidence of effectiveness from randomised controlled trials. Ageing Research Reviews. 2013;12(1):25362. doi: 10.1016/j.arr.2012.07.001. 
56. Basu S, Kwee TC, Surti S, Akin EA, Yoo D, Alavi A. Fundamentals of PET and PET/CT imaging. Annals of the New York Academy of Sciences. 2011;1228(1):1-18. doi: 10.1111/j.1749-6632.2011.06077.x.

57. Astrid Evan Der V, Egbert E, Adriaan Anthonius L. Positron emission tomography as a method for measuring drug delivery to tumors in vivo: the example of [11C]docetaxel. Frontiers in Oncology. 2013;3. doi: 10.3389/fonc.2013.00208.

58. Alavi A, Reivich M. Guest editorial: The conception of FDG-PET imaging. Seminars in Nuclear Medicine. 2002;32(1):2-5. doi: 10.1053/snuc.2002.29269.

59. Mosconi L, Tsui W, Herholz K, Pupi A, Drzezga A, Lucignani G, et al. Multicenter Standardized 18F-FDG PET Diagnosis of Mild Cognitive Impairment, Alzheimer's Disease, and Other Dementias. The Journal of Nuclear Medicine. 2008;49(3):390-8. doi: 10.2967/jnumed.107.045385.

60. Eduardo RZ, Maxime JP, Débora GS, Antoine L, Clotilde L, Hyoung-Ihl $\mathrm{K}$, et al. [18F]FDG PET signal is driven by astroglial glutamate transport. Nature Neuroscience. 2017;20(3). doi: 10.1038/nn.4492.

61. Stoessl AJ. Glucose utilization: still in the synapse. Nature Neuroscience. 2017;20(3):382. doi: 10.1038/nn.4513. 
62. Blass JP. Brain metabolism and brain disease: Is metabolic deficiency the proximate cause of Alzheimer dementia? Journal of Neuroscience Research. 2001;66(5):851-6. doi: 10.1002/jnr.10087.

63. Dodart JC, Mathis C, Bales KR, Paul SM, Ungerer A. Early regional cerebral glucose hypometabolism in transgenic mice overexpressing the V717F $\beta$-amyloid precursor protein. Neuroscience Letters. 1999;277(1):49-52. doi: 10.1016/S0304-3940(99)00847-2.

64. Adams JN, Lockhart SN, Li L, Jagust WJ. Relationships Between Tau and Glucose Metabolism Reflect Alzheimer's Disease Pathology in Cognitively Normal Older Adults. Cerebral Cortex. 2019;29(5):19972009. doi: 10.1093/cercor/bhy078.

65. Pooler AM, Polydoro M, Maury EA, Nicholls SB, Reddy SM, Wegmann $\mathrm{S}$, et al. Amyloid accelerates tau propagation and toxicity in a model of early Alzheimer's disease. Acta Neuropathologica Communications. 2015;3(1). doi: 10.1186/s40478-015-0199-x.

66. Mueller SG, Weiner MW, Thal LJ, Petersen RC, Jack CR, Jagust W, et al. Ways toward an early diagnosis in Alzheimer's disease: The Alzheimer's Disease Neuroimaging Initiative (ADNI). Alzheimer's \& 
Dementia: The Journal of the Alzheimer's Association. 2005;1(1):55-66. doi: 10.1016/j.jalz.2005.06.003.

67. Shaw L, Vanderstichele H, Knapik-Czajka M, Figurski M, Coart E, Blennow K, et al. Qualification of the analytical and clinical performance of CSF biomarker analyses in ADNI. Acta Neuropathologica. 2011;121(5):597-609. doi: 10.1007/s00401-011-0808-0.

68. Crane P, Carle A, Gibbons L, Insel P, Mackin R, Gross A, et al. Development and assessment of a composite score for memory in the Alzheimer's Disease Neuroimaging Initiative (ADNI). Brain Imaging and Behavior. 2012;6(4):502-16. doi: 10.1007/s11682-012-9186-z.

69. Tatsuoka MM. Graph Theory and Its Applications in Educational Research: A Review and Integration. Review of Educational Research. 1986;56(3):291-329. doi: 10.3102/00346543056003291.

70. Hubert L. Some applications of graph theory to clustering. Psychometrika. 1974;39(3):283-309. doi: 10.1007/BF02291704.

71. Mashaghi A, Ramezanpour A, Karimipour V. Investigation of a protein complex network. The European Physical Journal B - Condensed Matter and Complex Systems. 2004;41(1):113-21. doi: 10.1140/epjb/e200400301-0. 
72. Tarapata Z, Kasprzyk R. An Application of Multicriteria Weighted Graph Similarity Method to Social Networks Analyzing. IEEE; 2009. p. $366-8$.

73. Shah P, Ashourvan A, Mikhail F, Pines A, Kini L, Oechsel K, et al. Characterizing the role of the structural connectome in seizure dynamics. Brain : a journal of neurology. 2019;142(7):1955. doi: 10.1093/brain/awz125.

74. Lovejoy WS, Loch CH. Minimal and maximal characteristic path lengths in connected sociomatrices. Social Networks. 2003;25(4):333-47. doi: 10.1016/j.socnet.2003.10.001.

75. Duncan JW, Steven HS. Collective dynamics of 'small-world' networks. Nature. 1998;393(6684):440. doi: 10.1038/30918.

76. Schank T, Wagner D. Approximating clustering coefficient and transitivity. Journal of Graph Algorithms and Applications. 2005;9(2):265-75. doi: 10.7155/jgaa.00108.

77. Soffer S, Vazquez A. Network clustering coefficient without degreecorrelation biases. Physical Review E. 2005;71(5). doi: 10.1103/PhysRevE.71.057101. 
78. Saramaki J, Kivela M, Kaski K, Kertesz J. Generalizations of the clustering coefficient to weighted complex networks. arXivorg. 2006;75(2 Pt 2). doi: 10.1103/PhysRevE.75.027105.

79. Brandes U. A faster algorithm for betweenness centrality. The Journal of Mathematical Sociology. 2001;25(2):163-77. doi: 10.1080/0022250X.2001.9990249.

80. Freeman LC. A Set of Measures of Centrality Based on Betweenness. Sociometry. 1977;40(1):35-41. doi: 10.2307/3033543.

81. Arendt T. Synaptic degeneration in Alzheimer's disease. Acta Neuropathologica. 2009;118(1):167-79. doi: 10.1007/s00401-009-0536$\mathrm{X}$.

82. Takahashi RH, Capetillo-Zarate E, Lin MT, Milner TA, Gouras GK. Cooccurrence of Alzheimer's disease $\beta$-amyloid and tau pathologies at synapses. Neurobiology of Aging. 2010;31(7):1145-52. doi: 10.1016/j.neurobiolaging.2008.07.021.

83. Jack CR, Knopman D, Jagust W, Shaw L, Aisen PS, Weiner M, et al. Hypothetical model of dynamic biomarkers of the Alzheimer's pathological cascade. Lancet Neurology. 2010;9(1):119-28. doi: 10.1016/S1474-4422(09)70299-6. 
84. Braak H, Braak E. Neuropathological stageing of Alzheimer-related changes. Acta Neuropathologica. 1991;82(4):239-59. doi: 10.1007/BF00308809.

85. Tijms BM, Wink AM, de Haan W, van Der Flier WM, Stam CJ, Scheltens P, et al. Alzheimer's disease: connecting findings from graph theoretical studies of brain networks. Neurobiology of Aging. 2013;34(8):2023-36. doi: 10.1016/j.neurobiolaging.2013.02.020.

86. Ed B, Olaf S. Complex brain networks: graph theoretical analysis of structural and functional systems. Nature Reviews Neuroscience. 2009;10(3):186. doi: 10.1038/nrn2575.

87. Bassett DS, Bullmore E, Verchinski BA, Mattay VS, Weinberger DR, Meyer-Lindenberg A. Hierarchical organization of human cortical networks in health and schizophrenia. The Journal of neuroscience : the official journal of the Society for Neuroscience. 2008;28(37):9239-48. doi: 10.1523/JNEUROSCI.1929-08.2008.

88. Lynall M-E, Bassett DS, Kerwin R, McKenna PJ, Kitzbichler M, Muller U, et al. Functional connectivity and brain networks in schizophrenia. The Journal of neuroscience : the official journal of the Society for 
Neuroscience. 2010;30(28):9477-87. doi: 10.1523/JNEUROSCI.033310.2010.

89. Agosta F, Pievani M, Geroldi C, Copetti M, Frisoni GB, Filippi M. Resting state fMRI in Alzheimer's disease: beyond the default mode network. Neurobiology of Aging. 2012;33(8):1564-78. doi: 10.1016/j.neurobiolaging.2011.06.007.

90. Wang J, Zuo X, Dai Z, Xia M, Zhao Z, Zhao X, et al. Disrupted Functional Brain Connectome in Individuals at Risk for Alzheimer's Disease. Biological Psychiatry. 2013;73(5):472-81. doi: 10.1016/j.biopsych.2012.03.026.

91. He Y, Chen Z, Evans A. Structural insights into aberrant topological patterns of large-scale cortical networks in Alzheimer's disease. The Journal of neuroscience : the official journal of the Society for Neuroscience. 2008;28(18):4756-66. doi: 10.1523/JNEUROSCI.014108.2008.

92. Supekar K, Menon V, Rubin D, Musen M, Greicius MD. Network Analysis of Intrinsic Functional Brain Connectivity in Alzheimer's Disease (Analysis of Functional Brain Networks in AD). PLoS 
Computational Biology. 2008;4(6):e1000100. doi: 10.1371/journal.pcbi.1000100.

93. Yao Z, Zhang Y, Lin L, Zhou Y, Xu C, Jiang T. Abnormal Cortical Networks in Mild Cognitive Impairment and Alzheimer's Disease (Abnormal Cortical Networks in MCI and AD). PLoS Computational Biology. 2010;6(11):e1001006. doi: 10.1371/journal.pcbi.1001006.

94. Tijms BM, Möller C, Vrenken H, Wink AM, de Haan W, van der Flier WM, et al. Correction: Single-Subject Grey Matter Graphs in Alzheimer's Disease. PLoS ONE. 2013;8(9). doi: 10.1371/annotation/6a2e6405-ce1d-49e0-a88c-0017c680d597.

95. McKhann G, Drachman D, Folstein M, Katzman R, Price D, Stadlan EM. Clinical diagnosis of Alzheimer's disease: report of the NINCDSADRDA Work Group under the auspices of Department of Health and Human Services Task Force on Alzheimer's Disease. Neurology. 1984;34(7):939. doi: 10.1212/WNL.34.7.939.

96. Morris CJ. The Clinical Dementia Rating (CDR): Current version and scoring rules. Neurology. 1993;43(11):2412-4. doi: 10.1212/WNL.43.11.2412-a. 
97. Folstein MF, Folstein SE, McHugh PR. "Mini-mental state". A practical method for grading the cognitive state of patients for the clinician. Journal of psychiatric research. 1975;12(3):189-98. doi: 10.1016/00223956(75)90026-6.

98. Beheshti I, Demirel H, Matsuda H. Classification of Alzheimer's disease and prediction of mild cognitive impairment-to-Alzheimer's conversion from structural magnetic resource imaging using feature ranking and a genetic algorithm. Computers in Biology and Medicine. 2017;83:109-19. doi: 10.1016/j.compbiomed.2017.02.011.

99. Sled JG, Zijdenbos AP, Evans AC. A nonparametric method for automatic correction of intensity nonuniformity in MRI data. IEEE Transactions on Medical Imaging. 1998;17(1):87-97. doi: $10.1109 / 42.668698$.

100. Hosseini SMH, Hoeft F, Kesler SR. GAT: A Graph-Theoretical Analysis Toolbox for Analyzing Between-Group Differences in Large-Scale Structural and Functional Brain Networks (GAT: Graph Analysis Toolbox for Brain Networks). PLoS ONE. 2012;7(7):e40709. doi: 10.1371/journal.pone.0040709. 
101. Wijk vBCM, Stam CJ, Daffertshofer A. Comparing brain networks of different size and connectivity density using graph theory. PLoS One. 2010.

102. Barthélemy M. Betweenness centrality in large complex networks. The European Physical Journal B. 2004;38(2):163-8. doi: 10.1140/epjb/e2004-00111-4.

103. Bernhardt BC, Chen Z, He Y, Evans AC, Bernasconi N. GraphTheoretical Analysis Reveals Disrupted Small-World Organization of Cortical Thickness Correlation Networks in Temporal Lobe Epilepsy. Cerebral Cortex. 2011;21(9):2147-57. doi: 10.1093/cercor/bhq291.

104. Réka A, Hawoong J, Albert-László B. Error and attack tolerance of complex networks. Nature. 2000;406(6794):378. doi: $10.1038 / 35019019$.

105. Yong H, Zhang C, Gaolang G, Evans A. Neuronal Networks in Alzheimer's Disease. Los Angeles, CA: SAGE Publications; 2009. p. 333-50.

106. Schmahmann JD. Dysmetria of thought: clinical consequences of cerebellar dysfunction on cognition and affect. Trends in Cognitive Sciences. 1998;2(9):362-71. doi: 10.1016/S1364-6613(98)01218-2. 
107. Grady CL, Haxby JV, Horwitz B, Sundaram M, Berg G, Schapiro M, et al. Longitudinal study of the early neuropsychological and cerebral metabolic changes in dementia of the Alzheimer type. Journal of clinical and experimental neuropsychology. 1988;10(5):576-96. doi: 10.1080/01688638808402796.

108. Mentis MJ, Horwitz B, Grady CL, Alexander GE, Vanmeter JW, Maisog JM, et al. Visual cortical dysfunction in Alzheimer's disease evaluated with a temporally graded "stress test" during PET. American Journal of Psychiatry. 1996;153(1):32-40. doi: 10.1176/ajp.153.1.32.

109. Convit A, de Asis J, de Leon MJ, Tarshish CY, De Santi S, Rusinek H. Atrophy of the medial occipitotemporal, inferior, and middle temporal gyri in non-demented elderly predict decline to Alzheimer's disease 2 . Neurobiology of Aging. 2000;21(1):19-26. doi: 10.1016/S01974580(99)00107-4.

110. Ryan NS, Keihaninejad S, Shakespeare TJ, Lehmann M, Crutch SJ, Malone IB, et al. Magnetic resonance imaging evidence for presymptomatic change in thalamus and caudate in familial Alzheimer's disease. Brain. 2013;136(5):1399-414. doi: 10.1093/brain/awt065. 
111. Seeley WW, Crawford RK, Zhou J, Miller BL, Greicius MD. Neurodegenerative Diseases Target Large-Scale Human Brain Networks. Neuron. 2009;62(1):42-52. doi: 10.1016/j.neuron.2009.03.024.

112. de Haan W, van Der Flier WM, Wang H, Van Mieghem PFA, Scheltens P, Stam CJ. Disruption of Functional Brain Networks in Alzheimer's Disease: What Can We Learn from Graph Spectral Analysis of RestingState Magnetoencephalography? Brain Connectivity. 2012;2(2):45-55. doi: 10.1089/brain.2011.0043.

113. Hillebrand A, Barnes GR, Bosboom JL, Berendse HW, Stam CJ. Frequency-dependent functional connectivity within resting-state networks: An atlas-based MEG beamformer solution. NeuroImage. 2012;59(4):3909-21. doi: 10.1016/j.neuroimage.2011.11.005. 